\title{
Preparation and self-assembly of amphiphilic polylysine dendrons
}

\author{
Mirsharghi, Sahar; Knudsen, Kenneth D.; Bagherifam, Shahla; Nyström, Bo; Boas, Ulrik
}

Published in:

New Journal of Chemistry

Link to article, DOI:

10.1039/c5nj02690c

Publication date:

2016

Document Version

Publisher's PDF, also known as Version of record

Link back to DTU Orbit

Citation (APA):

Mirsharghi, S., Knudsen, K. D., Bagherifam, S., Nyström, B., \& Boas, U. (2016). Preparation and self-assembly of amphiphilic polylysine dendrons. New Journal of Chemistry, 40(4), 3597-3611.

https://doi.org/10.1039/c5nj02690c

\section{General rights}

Copyright and moral rights for the publications made accessible in the public portal are retained by the authors and/or other copyright owners and it is a condition of accessing publications that users recognise and abide by the legal requirements associated with these rights.

- Users may download and print one copy of any publication from the public portal for the purpose of private study or research.

- You may not further distribute the material or use it for any profit-making activity or commercial gain

- You may freely distribute the URL identifying the publication in the public portal

If you believe that this document breaches copyright please contact us providing details, and we will remove access to the work immediately and investigate your claim. 


\section{PAPER}

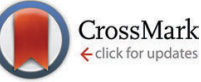

Cite this: New J. Chem., 2016, 40, 3597

Received (in Montpellier, France) 1st October 2015,

Accepted 15th February 2016

DOI: $10.1039 / c 5 n j 02690 c$

www.rsc.org/njc

\section{Preparation and self-assembly of amphiphilic polylysine dendrons $\dagger$}

\author{
Sahar Mirsharghi, ${ }^{a}$ Kenneth D. Knudsen, ${ }^{b}$ Shahla Bagherifam, ${ }^{c d}$ Bo Nyström ${ }^{c}$ and \\ Ulrik Boas*a
}

Herein, we present the synthesis of new amphiphilic polylysine dendrons with variable alkyl chain lengths $(\mathrm{C} 1-\mathrm{C} 18)$ at the $\mathrm{C}$-terminal. The dendrons were synthesized in moderate to quantitative yields by divergent solid-phase synthesis (SPS) employing an aldehyde linker. The self-assembling properties of the dendrons in aqueous solutions were studied by small angle neutron scattering (SANS) and dynamic light scattering (DLS). The self-assembling properties were influenced by the length of the alkyl chain and the generation number $(\mathrm{Gn})$. Increasing the temperature and concentration did not have significant impact on the hydrodynamic diameter, but the self-assembling properties were influenced by the $\mathrm{pH}$ value. This demonstrated the need for positively charged amines in the head groups for the successful formation of controlled self-assemblies. Dendrons having alkyl chains below C8 did not self-assemble. Well-defined micellar structures observed with SANS were formed with alkyl chain lengths above C12. Large structures detected with DLS for dendrons with alkyl chain lengths above C12 are ascribed to intermicellar aggregates stabilized by hydrophobic and electrostatic forces in accordance with the observed $\mathrm{pH}$ effect. Finally, the cytotoxicity of the dendrons was evaluated in mouse fibroblast $(\mathrm{NIH} / 3 \mathrm{~T} 3)$ and human embryonic kidney (HEK 293T) cells at 5, 10 and $20 \mu \mathrm{M}$ concentrations. The dendrons showed low cytotoxicity, displaying cell viability well above $80 \%$.

\section{Introduction}

Dendrimers and dendrons belong to a class of well-defined/ monodisperse hyperbranched molecules. The first molecular dendritic structures reported in the literature were poly(propylene imine) (PPI or POPAM) initially named "cascade" molecules by Buhleier et $a .^{1}{ }^{1}$ in 1978. However, it was Tomalia et al. ${ }^{2}$ who defined and named dendrimers in 1985 and described the synthesis of bigger structures, i.e., poly(amidoamine) (PAMAM) dendrimers. Independently around the same time Newkome et $a l^{3}$ reported the synthesis of similar macromolecules, poly(etheramide) (Arborol) dendrimers. The first peptide-based dendrimers and dendrons were developed by Denkewalter and co-workers in the early 1980s, and were composed of L-lysine as

\footnotetext{
${ }^{a}$ National Veterinary Institute, Technical University of Denmark (DTU), Bülowsvej 27, DK-1870 Frederiksberg C, Denmark. E-mail: uboa@vet.dtu.dk

${ }^{b}$ Department of Physics, Institute for Energy Technology, PO Box 40, N-2027 Kjeller, Norway

${ }^{c}$ Department of Chemistry, University of Oslo, PO Box 1033, Blindern N-0315, Oslo, Norway

${ }^{d}$ Department of Biology, University of Oslo, Blindernveien 31, N-0316 Oslo, Norway $\dagger$ Electronic supplementary information (ESI) available: Measured zeta potentials for G1 and G2 dendrons. IR data for four selected derivatized resins. Assigned NMR spectra $\left({ }^{1} \mathrm{H}-\mathrm{NMR},{ }^{13} \mathrm{C}-\mathrm{NMR}\right.$, DEPT-135 ${ }^{13} \mathrm{C}-\mathrm{NMR}$, HSQC and COSY) and UPLC-MS chromatograms are provided for all compounds. See DOI: 10.1039/c5nj02690c
}

the repeating monomer. ${ }^{4,5}$ Here, lysine has two amine functionalities available to serve as branching units. These structures were later revisited by Tam and co-workers, who constructed the well-defined multiple antigenic peptide (MAP) system that was originally intended for vaccines. MAPs are mainly based on polylysine. They have found additional use in other applications, e.g., diagnostics, drug delivery, and gene delivery. ${ }^{6-8}$ Dendrimers and dendrons are classified by their generation numbers $(\mathrm{G} n)$ according to the number $(n)$ of branching points of repeating units from the core, or for polylysine dendrons, from the C-terminal to the periphery ( $\mathrm{N}$-terminal). With each generation number, the number of surface functionalities will increase. The surface groups can be modified with the molecular motifs of interest giving dendrimers and dendrons the ability of multivalent display. This multivalency makes dendrimers and dendrons promising for a variety of biomedical applications. ${ }^{9-11}$ Recent studies on dendrons carrying a lipid tail have shown that they hold great promise as non-viral transfectants for genetic materials such as siRNA. ${ }^{12-14}$ Hence, the ability to form selfassembled structures may be an important feature when applying dendrimers as carriers for drugs and genes.

In the present study, we synthesized novel polylysine dendrons with different alkyl chain lengths at the C-terminal to investigate the self-assembly behavior as a function of the alkyl chain length and dendron generation. We hypothesized that the lipid functionality on the dendrons would induce self-assembly into micelle-like structures 
by mainly hydrophobic interactions. Further modification of the surface functional groups of these dendritic structures with relevant ligands for a multivalent presentation can increase receptor recognition and binding due to the "dendritic effect". 9 The aim of this work is to elucidate the self-assembling features of these new systems and to gain insight into their potential to form supermolecular structures. For this purpose, it is highly relevant that the self-assembling properties of unmodified dendrons are well understood.

\section{Results and discussion}

\section{Synthesis of polylysine dendrons}

Dendrons were synthesized by step-wise divergent solid-phase synthesis, using a backbone amide linker strategy (Scheme 1), ${ }^{15,16}$ which opens up the way for the preparation of dendrons with a large variety of C-terminal modifications. Here, a bisalkoxybenzaldehyde (4-(4'-formyl-3'-methoxyphenoxy) butanoic acid, FMPB) linker was attached to amino methylated polystyrene (AM PS) resin by an amide bond employing $N, N, N^{\prime}, N^{\prime}$-tetramethyl$O$-(1H-benzotriazol-1-yl)uronium hexafluorophosphate (HBTU) or

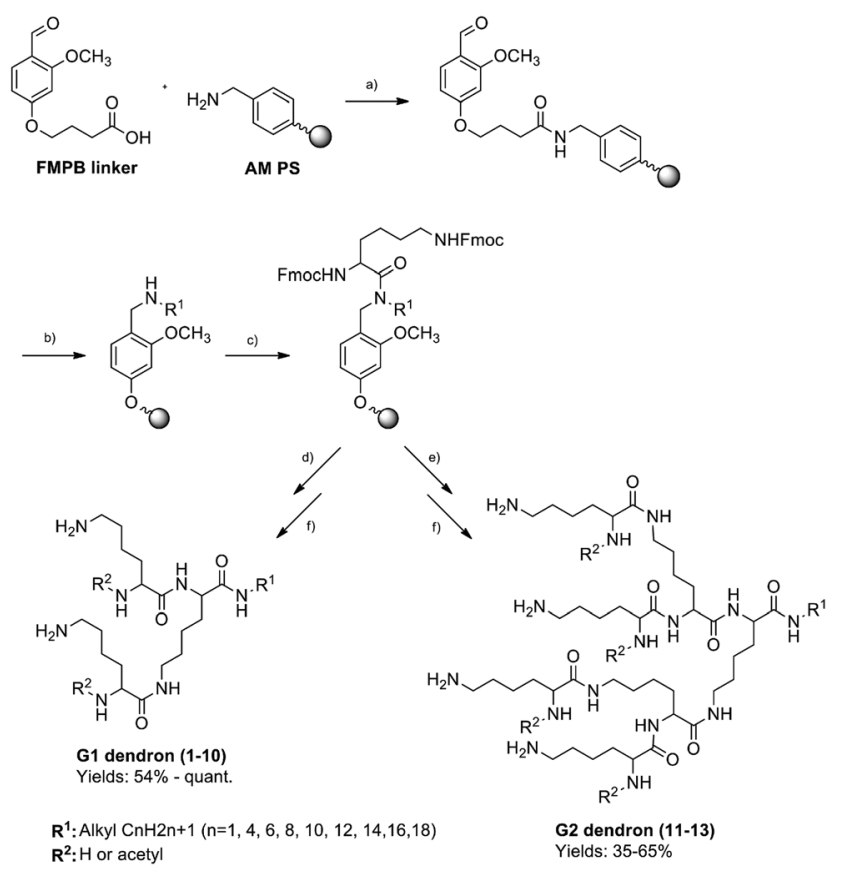

Scheme 1 Synthesis of G1 and G2 polylysine dendrons. (a) PyBOP or HBTU, DIPEA, NMP, rt, 22 h; (b) alkyl amine, $\mathrm{NaBH}_{3} \mathrm{CN}, 5 \% \mathrm{AcOH}, \mathrm{NMP}, \mathrm{rt}$, 20 h; (c) Fmoc-Lys(Fmoc)-OH, DIC, DCM/NMP (95: 5), rt, 20 h; (d) Fmoc deprotection: piperidine/NMP (1:4), rt, $3+30+20 \mathrm{~min}$; linkage of the second lysine group: Fmoc-Lys(Boc)-OH, PyBOP, DIPEA, NMP, rt, 3 h or until ninhydrin test negative; (e) Fmoc deprotection: piperidine/NMP (1: 4), rt, $3+30+20$ min; linkage of the second lysine group: Fmoc-Lys(Fmoc)$\mathrm{OH}$, PyBOP, DIPEA, rt, 3 h or until ninhydrin test negative; Fmoc deprotection: piperidine/NMP (1:4), rt, $3+30+20 \mathrm{~min}$; linkage of the third lysine group: Fmoc-Lys(Boc)-OH, PyBOP, DIPEA, rt, 3 h or until ninhydrin test negative; Fmoc deprotection: piperidine/NMP (1:4), rt, $3+30+20$ min; (f) $\mathrm{R}^{2}=\mathrm{H}$ : TFA/DCM (1:1), rt, $2 \mathrm{~h} ; \mathrm{R}^{2}=$ acetyl: $A c_{2} \mathrm{O} / \mathrm{DIPEA} / \mathrm{DCM}(10 / 5 / 85)$, rt, 3 h; TFA/DCM (1:1), rt, 2 h. (benzotriazol-1-yloxy)tripyrrolidinophosphonium hexafluorophosphate (PyBOP) as the coupling reagent together with $\mathrm{N}, \mathrm{N}$-diisopropylethylamine (DIPEA).

The completion of the reaction on the resin bead was monitored by infrared (IR) spectroscopy (attenuated total reflectance) showing new bands at approximately $1670 \mathrm{~cm}^{-1}(\mathrm{C}=\mathrm{O}$ stretch, amide $)$ and $1600 \mathrm{~cm}^{-1}$ ( $\mathrm{C}=\mathrm{O}$ stretch, aldehyde). Additionally, the reaction was monitored colorimetrically with 2,4-dinitrophenylhydrazine (DNPH) in sulfuric acid giving dark orange-red beads for resin-bound aldehyde groups according to the procedure by Shannon et al. ${ }^{17}$ Subsequently, reductive amination between the FMPB aldehyde group and an alkyl amine allowed a C-terminal modification by an alkyl chain of choice (Scheme 1). The reductive amination was carried out by employing sodium cyanoborohydride $\left(\mathrm{NaBH}_{3} \mathrm{CN}\right)$ in $\mathrm{N}$-methyl-2-pyrrolidone (NMP) under mild acidic conditions ( $5 \%$ acetic acid). The reaction completion was monitored by IR, which showed an increase in the $\mathrm{C}-\mathrm{H}$ stretch band around $2922 \mathrm{~cm}^{-1}$ and a decrease in the aldehyde band around $1610 \mathrm{~cm}^{-1} \cdot{ }^{18}$ This increase was more pronounced for dendrons modified with long chain alkyl amines at the C-terminal. Furthermore, the reaction was monitored by two complementary on-resin colorimetric methods: DNPH test (pale orange to yellow beads in accordance with a negative readout) and a newly developed secondary amine test, ${ }^{19,20}$ which gives orange-brown resin beads in the presence of resin-bound secondary amines. After this, acylation of the secondary amine on the resin by the first lysine residue was performed by Fmoc-Lys(Fmoc)-OH and $N, N^{\prime}$-diisopropylcarbodiimide (DIC) as the coupling reagent in a dichloromethane (DCM)/NMP mixture. An insoluble waxy $N, N^{\prime}$-diisopropylurea byproduct formed after 5 minutes and was removed after reaction completion by washing the resin thoroughly with NMP and $\mathrm{MeOH}$. Also here, the reaction completion was monitored colorimetrically giving a negative secondary amine test (colorless beads). In order to determine the final yield of the solid-phase synthesis, resin loadings were determined after the first lysine group had been added by measuring the UV-vis absorbance at $290 \mathrm{~nm}$ (dibenzofulvenepiperidine adduct released upon Fmoc cleavage) upon treatment of the resin-bound Fmoc-derivative with $20 \%$ piperidine in NMP. The loadings typically ranged from 0.14 to $0.35 \mathrm{mmol} \mathrm{g}^{-1}$.

Deprotection of the Fmoc-amines was conducted by the addition of a solution of $20 \%$ piperidine in NMP resulting in free primary amine groups for further reaction with the next lysine residue. The successful deprotection and subsequent coupling process were monitored colorimetrically by ninhydrin test (positive: purple-blue supernatant, negative: pale grey/ yellow supernatant). ${ }^{21,22}$ The second lysine was attached by employing PyBOP as the coupling reagent in NMP, and the reaction completion was again monitored by ninhydrin test. Either Fmoc-Lys(Boc)-OH or Fmoc-Lys(Fmoc)-OH was used as the second lysine depending on the product being a G1 or G2 dendron, respectively. For the G2 dendron the coupling was repeated with the attachment of Fmoc-Lys(Boc)-OH after the deprotection step. Cleavage of the dendron from the resin was carried out with a 1:1 mixture of trifluoroacetic acid (TFA) and DCM giving the dendrons in moderate to good yields and high 
Table 1 Synthesized G1 and G2 dendrons

\begin{tabular}{lll}
\hline$\#$ & Compound & Yield $^{a}(\%)$ \\
\hline 1 & G1-C1 & 70 \\
2 & G1-C8 & 64 \\
3 & G1-C12 & 54 \\
4 & G1-C14 & Quantitative \\
5 & G1-C16 & 79 \\
6 & G1-C18 & Quantitative \\
7 & G1(acetyl)-C4 & 81 \\
8 & G1(acetyl)-C6 & 73 \\
9 & G1(acetyl)-C10 & Quantitative \\
10 & G1(acetyl)-C16 & 72 \\
11 & G2-C1 & 35 \\
12 & G2-C12 & 43 \\
13 & G2-C16 & 65 \\
$a$ & &
\end{tabular}

crude purity (see Table 1 for compound numbers and yields). To obtain dendrons having partially acetylated N-terminal amines, the Fmoc groups were removed and the dendrons having Bocylated side chain amines were treated with $\mathrm{Ac}_{2} \mathrm{O} / \mathrm{DIPEA} /$ DCM (10/5/85) prior to the release of the dendron from the resin by treatment with acid (Scheme 1).

The chemical structure of the synthesized dendrons was confirmed by ${ }^{1} \mathrm{H}-\mathrm{NMR},{ }^{13} \mathrm{C}-\mathrm{NMR}$, DEPT-135 ${ }^{13} \mathrm{C}-\mathrm{NMR}$, COSY and HSQC and the molecular weight was determined by UPLC-MS. An example of typical 1D and 2D NMR spectra can be seen for the G1-C8 dendron in Fig. $1 .^{23}$

\section{Self-assembly probed by small angle neutron scattering}

In order to obtain information on a mesoscopic length scale and to reveal the structure of the self-assembled species, small angle neutron scattering (SANS) was performed on synthesized G1-C1/C8/C12/C14/C16/C18, G1(acetyl)-C10/C16 and G2-C1/C12/C16 dendrons. Dendron concentrations of 9, 18 and $27 \mathrm{mM}$ were chosen to observe the effect of concentration on the size and shape of the self-assembled entities. It should be noted that the considered concentrations are well above the critical aggregation concentration (cac) for the studied systems. To illustrate the scattering behavior, we show here initially SANS scattering data for two selected dendron types, G1-C16 and G1-C18, in aqueous solution (Fig. 2).

Strong interparticle interactions are observed in the form of a correlation peak at intermediate $q$-values (around $q=0.045 \AA^{-1}$ ), corresponding to an interparticle distance $d_{\mathrm{i}}=2 \pi / q$ around $140 \AA$ (14 nm). This allows for some separation between the micelles, since the overall diameter of these micelles was found to be ca. $5 \mathrm{~nm}$ (Table 2). The dynamic light scattering (DLS) results discussed below reveal that for these dendrons also some larger clusters (in the range around $100 \mathrm{~nm}$ ) are formed in solution (cf. Fig. 9). These entities have, however, a size outside the range for the SANS detection, and will not contribute to the characteristic micellar SANS pattern that we have seen, apart from a possible weak contribution at the lowest $q$-range where the SANS data are noisy. The SANS measurements therefore probe the micellar range only, but this does not exclude the presence of larger structures that we in some cases observe by DLS.
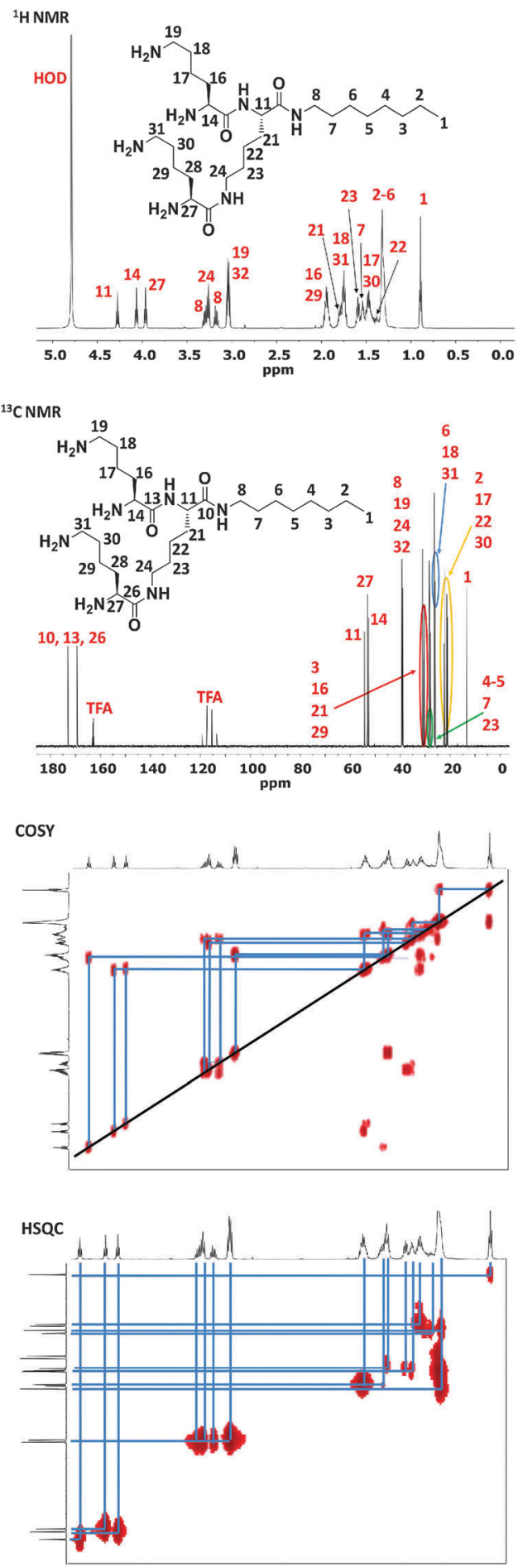

Fig. 1 Top: Assigned 1D NMR spectra of the G1-C8 dendron in $\mathrm{D}_{2} \mathrm{O}$ $\left({ }^{1} \mathrm{H}-\mathrm{NMR} 600 \mathrm{MHz}\right.$ and $\left.{ }^{13} \mathrm{C}-\mathrm{NMR} 151 \mathrm{MHz}\right)$. Bottom: 2D NMR spectra of the G1-C8 dendron showing the coupling between ${ }^{1} \mathrm{H}-{ }^{1} \mathrm{H}$ nuclei (COSY) and ${ }^{13} \mathrm{C}-{ }^{1} \mathrm{H}$ nuclei (HSQC).

The data can be well portrayed by a core-shell particle model when a repulsive interaction term between the particles is included. For interacting spherical entities the scattered intensity can be expressed through the product of a form factor $P(q)$ and an interaction term $S(q)$ (structure factor). The following form factor 


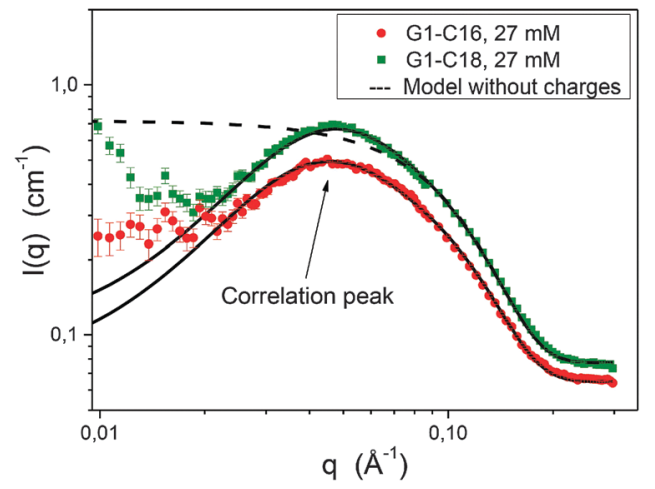

Fig. 2 SANS profiles of the G1-C16 and G1-C18 dendrons in aqueous solutions at a concentration of $27 \mathrm{mM}$. Continuous lines are fits with a spherical core-shell model (see text). The dashed line for G1-C18 represents the same core-shell model, but without charges.

Table 2 Fitted parameters for the G1-C16 and G1-C18 micelles. Error in the sizes is $\pm 0.4 \AA$, in the charge $\pm 0.5 \mathrm{e}$, and in the aggregation number \pm 5

\begin{tabular}{lllllll}
\hline & $\begin{array}{l}\text { Concentration } \\
{[\mathrm{mM}]}\end{array}$ & $\begin{array}{l}R \text { (core) } \\
{[\AA]}\end{array}$ & $\begin{array}{l}\text { Thickness } \\
(\text { shell })[\AA]\end{array}$ & $\begin{array}{l}R \text { (total) } \\
{[\AA]}\end{array}$ & $\begin{array}{l}\text { Charge } \\
(+)\end{array}$ & $N(\mathrm{agg})$ \\
\hline G1-C16 & 27 & 12.0 & 12.3 & 24.3 & 7.9 & 33 \\
G1-C18 & 27 & 12.8 & 11.9 & 24.7 & 8.0 & 35
\end{tabular}

$P(q)$, representing a spherical core-shell structure, was employed in the analysis: ${ }^{24}$

$$
P(q)=\frac{\text { scale }}{V_{\mathrm{s}}}\left[\begin{array}{l}
3 V_{\mathrm{c}}\left(\rho_{\mathrm{c}}-\rho_{\mathrm{s}}\right) \frac{\left[\sin \left(q r_{\mathrm{c}}\right)-q r_{\mathrm{c}} \times \cos \left(q r_{\mathrm{c}}\right)\right]}{\left(q r_{\mathrm{c}}\right)^{3}} \\
+3 V_{\mathrm{s}}\left(\rho_{\mathrm{s}}-\rho_{\mathrm{solv}}\right) \frac{\left[\sin \left(q r_{\mathrm{s}}\right)-q r_{\mathrm{s}} \times \cos \left(q r_{\mathrm{s}}\right)\right]}{\left(q r_{\mathrm{s}}\right)^{3}}
\end{array}\right]^{2}+\mathrm{bkg}
$$

Here $V_{\mathrm{c}}$ is the volume of the core while $V_{\mathrm{s}}$ is the volume of the shell. The parameter $r_{\mathrm{s}}$ is the outer radius of the shell (with respect to the particle center) and $r_{\mathrm{c}}$ that of the core, while $\rho_{\mathrm{c}}, \rho_{\mathrm{s}}$ and $\rho_{\text {solv }}$ are the scattering length densities of the core, shell, and solvent, respectively. In the modelling, $\rho_{\text {solv }}$ and $\rho_{\mathrm{c}}$ were set to the theoretical values for the scattering length density of $\mathrm{D}_{2} \mathrm{O}\left(\rho_{\text {solv }}=\right.$ $\left.6.3 \times 10^{-6} \AA^{-2}\right)$ and the alkyl chain $\left(\rho_{\mathrm{c}}=-0.34 \times 10^{-6} \AA^{-2}\right)$, respectively. The parameter $\rho_{\mathrm{s}}$ corresponds to the average scattering length density of the atoms in the dendron head groups, together with the water molecules located inside the shell, and was adjusted for a best fit to the data. The value of $\rho_{\mathrm{s}}$ was approximately $4 \times 10^{-6} \AA^{-2}$, indicating that the shell was significantly hydrated. The term scale is a factor proportional to the concentration, and bkg is the background due to incoherent scattering. To illustrate the significance of electrostatic interactions, the dashed line for G1-C18 in Fig. 2 represents the same core-shell model without charges. It is obvious that this model does not portray the data well and this emphasizes the importance of including charges in the model for a good description of the data.

Since these micelles are likely to be charged, due to the presence of amine groups, the structure factor employed was that corresponding to particles with Coulomb interaction, as developed by Hayter and Penfold. Below the structure factor is written in dimensionless form, where $K=q \sigma$, with $\sigma$ being the diameter of the spherical particle: ${ }^{25}$

$$
S(K)=\frac{1}{1-24 \eta a(K)}
$$

where $\eta=\pi n \sigma^{3} / 6$ is the volume fraction ( $n$ being the particle number density). The parameter $a(K)$ is a sum of polynomial terms (not shown here), which includes the effect of particle charge and counterions, as well as that of possible screening from additional ions in the solution.

Table 2 shows the extracted values for the core size and shell thickness using this model. The core radius is found to be $12 \AA$ for the G1-C16 micelle, and in the same range (12.8 $\mathrm{A})$ for the G1-C18 micelle. Even though the difference is small, and within the error level, one may in fact expect a slightly larger core radius for G1-C18 due to the longer alkyl chain (two more C atoms) that has to be accommodated into the micellar core.

The overall size of the micelles is found to be the same (within errors), i.e., about $25 \AA$ or $2.5 \mathrm{~nm}$ radius, corresponding to a diameter of $c a .5 \mathrm{~nm}$. When compared with the average distance between the micelles found earlier, $c a .14 \mathrm{~nm}$, we see that the surfaces of two neighboring micelles are separated by a distance of roughly two micellar diameters.

The aggregation number $N(\mathrm{agg})$, i.e., the number of dendron molecules taking part in each micelle, has also been estimated for these systems, as shown in Table $2 . N(\mathrm{agg})$ can be calculated based on the volume of one micelle, given by $(4 / 3) \pi R^{3}$, where $R$ is the total radius listed in Table 2, divided by the volume of one dendron molecule. The latter is given by $M_{\mathrm{w}} /\left(N_{\mathrm{A}} \cdot \rho\right)$, where $M_{\mathrm{W}}$ is the weight average molecular weight, $N_{\mathrm{A}}$ is Avogadro's constant $\left(6.02 \times 10^{23} \mathrm{~mol}^{-1}\right)$, and $\rho$ is the density $\left(\mathrm{g} \mathrm{cm}^{-3}\right)$. When calculating $M_{\mathrm{w}}$ one must take into account that for the G1 dendrons with four free amine groups, a minimum of four TFA molecules (each with $M_{\mathrm{w}}$ of $114.02 \mathrm{~g} \mathrm{~mol}^{-1}$ ) will be directly associated with the dendron. For other dendrons employed, such as the G1(acetyl)-C16 and Lys-C16 having two free amine groups (see later), one should take into account a minimum of two TFA and $\mathrm{HCl}$ molecules, respectively. For the estimate of $N(\mathrm{agg})$ we have assumed the number of associated TFA or $\mathrm{HCl}$ molecules being equal to the minimum number mentioned above, and furthermore employed the same density $\left(1 \mathrm{~g} \mathrm{~cm}^{-3}\right)$ for the different dendrons. We note that the aggregation numbers observed for G1-C16 and G1-C18 are $33 \pm 5$ and $35 \pm 5$ and they are significantly smaller than that of micelles of SDS having a radius of about $2 \mathrm{~nm}$ and an aggregation number of $c a .60 .^{26}$ This difference is expected because of the bulky head groups of the dendrons reported in this paper.

We also find that there is a charge on the micelles, corresponding to $c a .7$ charge units. This charge is due to protonated amines in the head groups of the dendrons. Since the head group is the same (G1) for the two dendrons above, it is also reasonable that the effective charge is found to be nearly the same in the two cases. This charge is responsible for quite a strong repulsion between the micelles, reflected in the structure factor $S(q)$, and leading to the depression of the curve to the left 
side of the correlation peak. As an illustration of the effect of charges on the scattering profile, the pattern of the same core-shell model without any charges present (all other parameters unchanged) is also shown (Fig. 2). We observe in Fig. 2 that the scattering signal tends to rise again at the lowest $q$-values and the upturn is strongest for the dendron (G1-C18) with the longest alkyl chain. This is most likely due to some large aggregates also present in the sample (cf. discussion of the DLS data below), and this part of the pattern (below $q=0.15 \AA^{-1}$ ) was not included in the fitting.

The charges present in the head groups of these dendrons are expected to have an influence on the stability of the created micelles, hindering the tendency to intermicellar aggregation. However, one may expect that these charges also can have an effect on the ability of the dendrons to assemble into micelles, due to Columbic repulsion that will occur between the head groups in the micellar shell. We therefore synthesized a variant of G1-C16 having less charges, G1(acetyl)-C16, with two acetylated amines and two free amines in the head group to compare the results with the SANS data on G1-C16 shown above, having four free amino groups in the head group. This turned out to considerably affect the SANS pattern, indicating an even stronger tendency to micelle formation. From a visual inspection of the results obtained (Fig. 3) we find that the characteristic micellar scattering pattern is significantly more developed for the less positively charged compound G1(acetyl)-C16 than for G1-C16. The intensity is higher (for the same concentration) and the correlation peak is narrower, demonstrating a well-defined average distance between similar entities (micelles).

We argue that the difference in behavior between the two dendrons is due to the stronger electrostatic repulsion within the corona of the micelles from compound G1-C16 that has four positively charged amine groups. This additional repulsion acts as a force working against the self-assembly of sufficient dendron molecules to create the most stable micelles. The SANS data on G1(acetyl)-C16 were also fitted using the spherical core-shell model described earlier, with the results shown in Fig. 3C. Table 3 shows the result of the fitting for this system. The radius of the core and the thickness of the shell of the micelle, as well as the extracted value for the micellar charge, are listed.

It is found that both the core radius (13-17 $⿱$ ) $)$ and the total radius (25-28 $\mathrm{A}$ ) of the micelles increase slightly with increasing concentration. When comparing the core radius obtained earlier for the G1-C16 micelle (Table 2) with the value for G1(acetyl)-C16 at the same concentration $(27 \mathrm{mM})$, we find a larger size for the latter (ca. 16 vs. $12 \AA$ ), whereas the thickness of the shell is nearly the same, and practically constant (roughly $12 \AA$ ) for the G1(acetyl)-C16 micelles over the concentration range considered in this study.

The size of the core radius is compatible with the expected size of an extended C16 chain, and the shell size matches with the approximate size of the head groups (roughly $10 \AA$ ). The slight expansion of the micelle size with increasing concentration is an indication that more unimers (dendrons) are incorporated into each micelle. This is reflected in the estimated aggregation number, and is also consistent with the finding that the effective charge density of the shell increases as the dendron concentration increases.
A

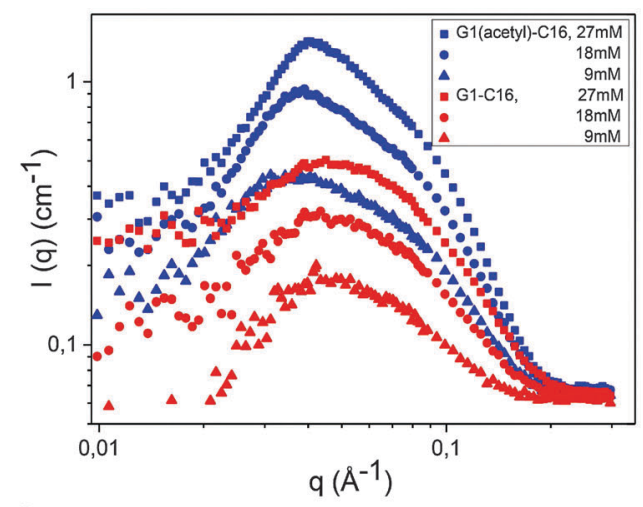

B

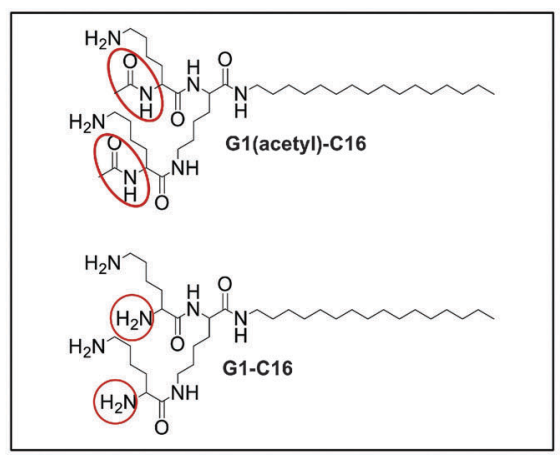

C

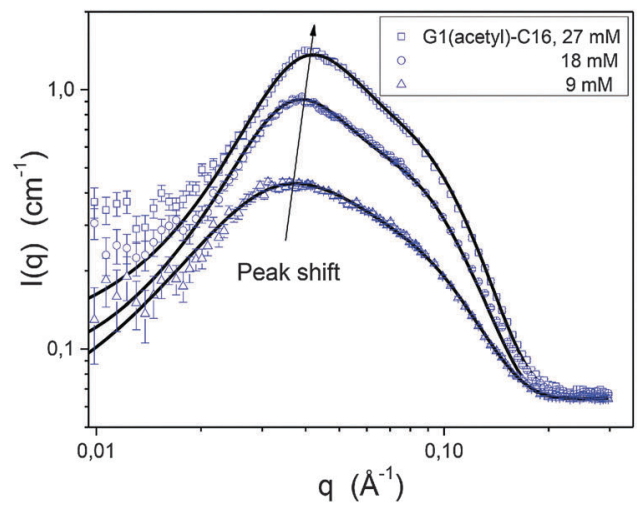

Fig. 3 (A) Comparison of SANS profiles from the two different G1-C16 dendrons, with 4 amine groups (G1-C16) and 2 amine groups (G1(acetyl)C16). (B) The molecular structures of the two different G1-C16 dendrons with head group differences marked by red circles. (C) SANS profiles of the G1(acetyl)-C16 dendron fitted with a spherical core-shell model.

Table 3 Fitted parameters for the G1(acetyl)-C16 micelles. The error in the sizes is $\pm 0.2 \AA$ and in the charge $\pm 0.5 \mathrm{e}$

\begin{tabular}{|c|c|c|c|c|c|c|}
\hline & $\begin{array}{l}\text { Concentration } \\
{[\mathrm{mM}]}\end{array}$ & $\begin{array}{l}R(\text { core }) \\
{[\AA]}\end{array}$ & $\begin{array}{l}\text { Thickness } \\
\text { (shell) }[\AA]\end{array}$ & $\begin{array}{l}R \text { (total) } \\
{[\AA]}\end{array}$ & $\begin{array}{l}\text { Charge } \\
(+)\end{array}$ & $N$ (agg) \\
\hline G1(acetyl)- & 9 & 13.4 & 12.5 & 25.9 & 10 & 47 \\
\hline \multirow[t]{2}{*}{ C16 } & 18 & 15.7 & 11.7 & 27.4 & 15 & 55 \\
\hline & 27 & 16.7 & 11.3 & 28.0 & 16 & 59 \\
\hline
\end{tabular}

The position of the correlation peak in Fig. 3C is seen to move to slightly higher $q$-values with increasing concentration, 
demonstrating that the average distance between the micelles becomes smaller due to the formation of more micelles. Thus the main effect of concentration increase for this system is the formation of additional micelles, with just a slight simultaneous increase in the micellar size.

We have now demonstrated the behavior and fitting procedure for some stable micellar structures, and to achieve that dendrons with a relatively long alkyl chain were selected. However, we also looked at the effect of different lengths of the alkyl chain, and found that there is a certain critical length necessary for micelles of these dendrons to be formed. SANS results for G1 dendrons with three different lengths of the alkyl chain and at various concentrations are depicted in Fig. 4A, and a visual inspection of the data gives important information about these systems.

For the sample with the shortest alkyl chain shown here (G1-C14), the correlation peak is virtually absent at the lowest concentration, but appears and is strengthened as the concentration increases. This behavior is consistent with the hypothesis that more molecules facilitate self-assembly and thereby favor the formation of core-shell like structures. Correlation peaks are seen to exist at all concentrations for G1-C16 (as commented earlier), reflecting the formation of organized spherical core-shell micelles, although this effect was found to be even stronger for G1(acetyl)-C16. This behavior is also found to be the case for G1-C18 at the three concentrations employed (Fig. 4A). These findings show that when the alkyl tails are sufficiently long, regular structures can emerge even at low concentration, and a longer alkyl chain (with the head group unchanged) enhances the formation of stable micelles. On the other hand, for the C14 alkyl chain, a higher concentration is needed to develop micellelike structures. As mentioned before ( $c f$. Fig. 2), although there is significant noise in the SANS data at low $q$-values, one can identify a significant upturn in the scattered intensity at low $q$ for most of the samples, indicating the existence of also some large structures in the system (see the discussion below on DLS results).

Furthermore, a comparison between two generations of dendrons, G1 and G2, was made. This is shown in Fig. 4C at a concentration of $c a .20 \mathrm{mg} \mathrm{mL}{ }^{-1}$. In addition, the SANS scattering profiles for dendrons with the shortest alkyl chain, G1-C1 and G2-C1, are also shown in the figure. These patterns are observed to be very similar and indicate that no regular micelle structures are formed, but the strong upturn at low $q$ may signalize the presence of large irregular aggregates. It should be mentioned that patterns for other alkyl chain lengths, G1-C8/C12 and G1(acetyl)-C10, were also collected (not shown here), but were practically overlapping with those of G1-C1, thus presenting no indication of micelle formation. When the data for G1-C16 and G2-C16 are compared, it is noticed that the SANS scattering profile for G2-C16 displays quite a different behavior than that of the former sample. For G2-C16 a plateau region is observed at intermediate $q$-values,
A

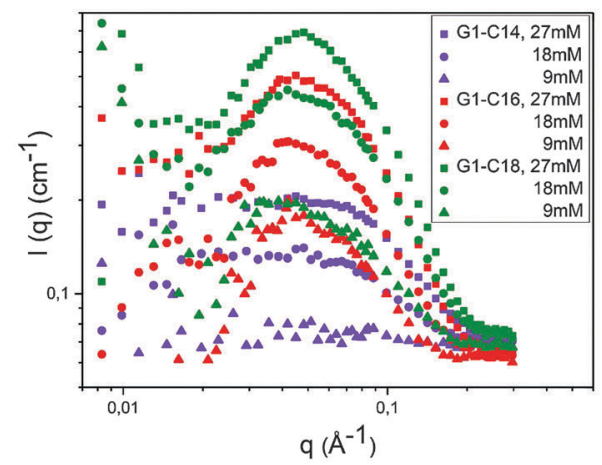

C

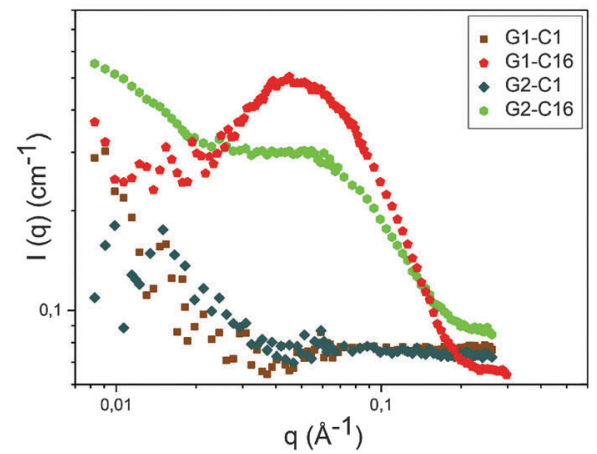

B

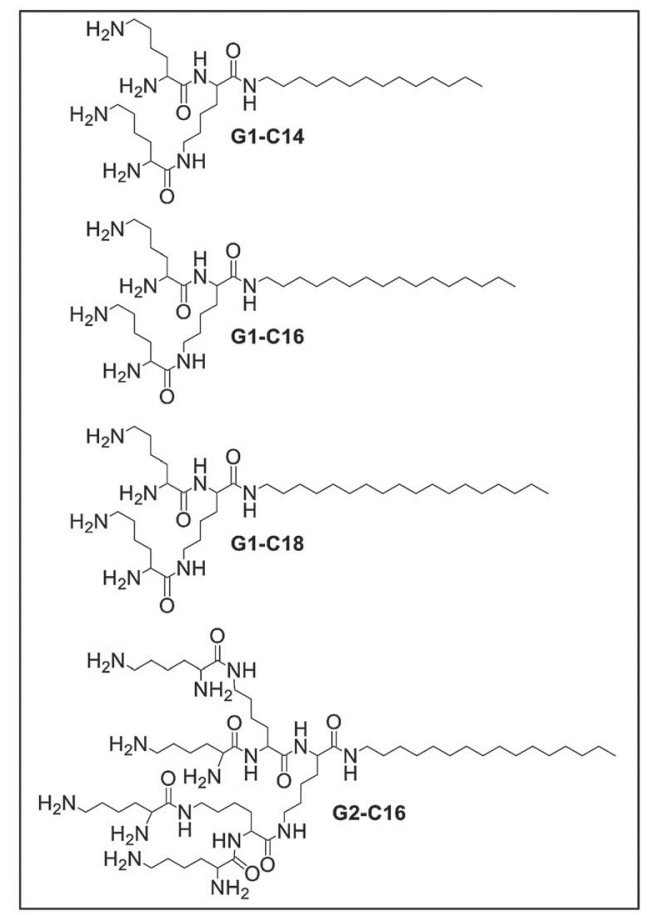

Fig. 4 (A) SANS profiles for G1-C14, G1-C16 and G1-C18 at different concentrations. (B) The molecular structures of G1 dendrons shown with different alkyl chain lengths (C14, C16 and $\mathrm{C} 18)$ and the G2-C16 dendron. (C) SANS profiles of G1 and G2 dendrons with variable C-terminal alkyl tails at a concentration of about $20 \mathrm{mg} \mathrm{mL}^{-1}$. 
followed by a strong upturn of the scattered intensity at low $q$. This clearly suggests the presence of large clusters in the solution, and indicates that the larger head groups in the G2 dendrons (compared to G1) may obstruct the formation of regular micelles. It is reasonable to assume that the efficiency of forming micelles should depend on the size and population of both polar and non-polar groups, lysine head group and alkyl chain, respectively. Thus for a moderate alkyl length (e.g., C16 as here), the size of the head group can easily become too large for efficient packing in the micellar corona.

Based on the previous observation of the effect of the head group, we also decided to investigate another variant of the C16-type dendron, namely Lys-C16 where the G1 head group (three lysine groups) has been replaced by an even smaller unit (one lysine group). This was in order to see if reducing the head group even further had any effect on the micelle formation. In Fig. 5, the SANS profiles for solutions of Lys-C16 are shown. The characteristic features are similar to those of the G1(acetyl)-C16 system (Fig. 3), with strong micellar scattering of nearly the same magnitude, being more accentuated than for the G1-C16 dendron. The same core-shell model as employed earlier was used in fitting these data, and the results are shown in Table 4.

Lys-C16 and G1(acetyl)-C16 are quite different in terms of size of the head group, but comparable with respect to charge, with both having two amines available for protonation, instead of the four amines in G1-C16. This is a strong indication that charge density is highly important with respect to micelle formation. This comes in addition to the earlier observation that for a given charge the size of the head group can work against the formation of stable micelles. Also for Lys-C16 more unimers are located in the micelle as the concentration increases.

A comparison of the results of the fitting for the Lys-C16 and the G1(acetyl)-C16 systems discloses (apart from the lowest concentration, where the data quality is moderate) that the thickness of the shell for Lys-C16 is slightly smaller than that for G1(acetyl)-C16. This is reasonable taking into account that Lys-C16 has a marginally smaller head group. We also see that the aggregation number has now increased considerably with

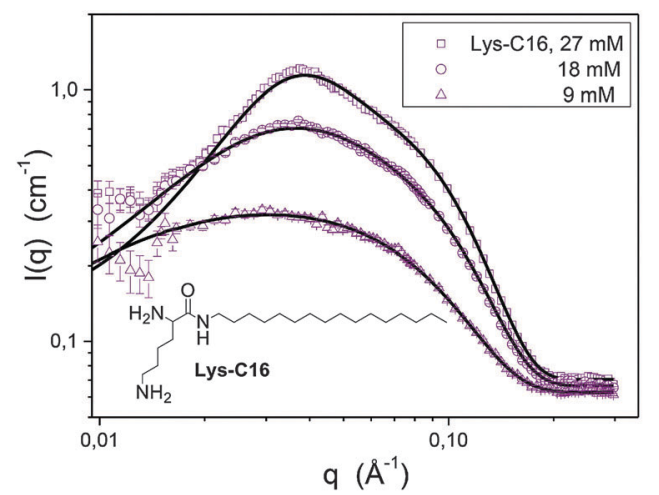

Fig. 5 SANS profiles of Lys-C16 (see the molecular structure inseted below the curves) in aqueous solutions at different concentrations. The curves are well fitted with a spherical core-shell model of charged micelles.
Table 4 Fitted parameters for the Lys-C16 micelles. The error in the radii is $\pm 0.2 \AA$ and in the charge $\pm 0.5 \mathrm{e}$

\begin{tabular}{|c|c|c|c|c|c|c|}
\hline & $\begin{array}{l}\text { Concentration } \\
{[\mathrm{mM}]}\end{array}$ & $\begin{array}{l}R \text { (core) } \\
{[\AA]}\end{array}$ & $\begin{array}{l}\text { Thickness } \\
\text { (shell) [̊] }\end{array}$ & $\begin{array}{l}R \text { (total) } \\
{[\AA]}\end{array}$ & $\begin{array}{l}\text { Charge } \\
(+)\end{array}$ & $N$ (agg) \\
\hline \multirow[t]{3}{*}{ Lys-C16 } & 9 & 13.5 & 14.4 & 27.9 & 4 & 124 \\
\hline & 18 & 17.9 & 10.7 & 28.6 & 7 & 133 \\
\hline & 27 & 18.6 & 10.5 & 29.1 & 12 & 140 \\
\hline
\end{tabular}

respect to the G1-type dendrons, most likely since the smaller Lys head group reduces the steric hindrance in the micellar corona, thus allowing for the hydrophobic interaction between the alkyl chains to assemble more dendrons in each micelle. Overall, the results for solutions of these dendrons demonstrate that both systems form spherical core-shell micelles with a total diameter between 5 and $6 \mathrm{~nm}$, where C16 alkyl chains constitute the core and the head group designs the shell.

\section{Self-assembly probed by dynamic light scattering}

The effects of the C-terminal alkyl group on the self-assembling properties of the G1 and G2 dendrons were investigated by DLS to gain insight into the sizes and structures formed in the self-assembly process on a more global dimensional scale than SANS. The influence of temperature, $\mathrm{pH}$ and concentration on the behavior of these systems was examined to obtain detailed information on the effects of these parameters on dynamics and structure.

Since our ultimate aim is to employ the synthesized polylysine dendrons for drug delivery purposes, four relevant temperatures were chosen: $8{ }^{\circ} \mathrm{C}$ (refrigerated storage conditions), $25{ }^{\circ} \mathrm{C}$ (shelf storage at rt), $37{ }^{\circ} \mathrm{C}$ (physiological) and $42{ }^{\circ} \mathrm{C}$ (hyperthermic case). We chose to investigate three different $\mathrm{pH}$ values: $\mathrm{pH} 5$ (present in lysosomes and tumors), $\mathrm{pH} 7.4$ (physiological conditions) and pH 9 to understand the behavior of the polylysine dendrons under basic conditions. To obtain these $\mathrm{pH}$ values, a phosphate buffered saline (PBS) solution was employed and the $\mathrm{pH}$ adjusted with $0.1 \mathrm{M} \mathrm{HCl}$ or $0.1 \mathrm{M} \mathrm{NaOH}$. Dendron concentrations of 4, 9 and $18 \mathrm{mM}$ were chosen to see whether an alteration in concentration may lead to changes in the size of the self-assembled entities.

In solutions of dendrons and other solutes, the experimentally recorded intensity autocorrelation function $g^{2}(q, t)$ is directly linked to the theoretically amenable first-order electric field autocorrelation $g^{1}(q, t)$ through the Siegert ${ }^{27}$ relationship $g^{2}(q, t)=1+B\left|g^{1}(q, t)\right|^{2}$, where $B(\leq 1)$ is an instrumental parameter. The wave vector is determined by $q=(4 \pi n / \lambda) \sin (\theta / 2)$, where $\lambda$ is the wavelength of the incident light in vacuum, $\theta$ is the scattering angle, and $n$ is the refractive index of the medium.

For most dendrons, the decay of the correlation functions generally showed a unimodal behavior and was fitted by a stretched exponential with only one relaxation mode:

$$
g^{1}(t)=A \exp \left[-\left(\frac{t}{\tau_{\mathrm{e}}}\right)^{\beta}\right]
$$

Here $A$ is the amplitude of the relaxation mode, $\tau_{\mathrm{e}}$ is the effective relaxation time, and $\beta(0 \leq \beta \leq 1)$ is a measure of the distribution of relaxation times. The average relaxation time 
is given by $\tau=\left(\tau_{\mathrm{e}} / \beta\right) \Gamma(1 / \beta)$, where $\Gamma(1 / \beta)$ is the gamma function of $\beta^{-1}$.

For the G1-C14/C16/C18 dendrons, the relaxation process was found to be more complex because large clusters are formed. In this case, bimodal decay is observed and the correlation functions are well portrayed by the sum of two stretched exponentials:

$$
g^{1}(t)=A_{\mathrm{f}} \exp \left[-\left(\frac{t}{\tau_{\mathrm{fe}}}\right)^{\beta_{\mathrm{f}}}\right]+A_{\mathrm{s}} \exp \left[-\left(\frac{t}{\tau_{\mathrm{se}}}\right)^{\beta_{\mathrm{s}}}\right]
$$

where $A_{\mathrm{f}}+A_{\mathrm{s}}=1$. The subscripts $\mathrm{f}$ and $\mathrm{s}$ of the amplitudes denote parameters for the fast and slow relaxation mode, respectively.

The correlation functions were analyzed by a non-linear fitting algorithm obtaining best-fit values for the relaxation times and stretched exponentials in eqn (3) or eqn (4). The relaxation time $\tau$ is always diffusive and therefore $q^{2}$ dependent. The relaxation time is related to the mutual diffusion coefficient $D$ by $\tau^{-1}=D \cdot q^{2}$ and the apparent hydrodynamic diameter $d_{\mathrm{h}}$ assuming spherical structures can be calculated via the StokesEinstein relationship:

$$
d_{\mathrm{h}}=\frac{k_{\mathrm{B}} T}{3 \pi \eta D}
$$

where $k_{\mathrm{B}}$ is the Boltzmann constant, $T$ is the absolute temperature, and $\eta$ is the viscosity of the medium.

DLS was employed to investigate the concentration effects on the self-assembling properties of the G2-C12 and G2-C16 dendrons, and the results indicate that the concentration did not have any notable effect on the sizes of the aggregates. The hydrodynamic diameters $d_{\mathrm{h}}$ were found to be $95 \mathrm{~nm}$ and $65 \mathrm{~nm}$, respectively (Fig. 6). In these cases the correlation functions could be well-described by a single stretched exponential (eqn (3)). However, the sizes of the species are outside the $q$-window probed in our SANS experiments.

These large hydrodynamic diameters observed by DLS are attributed to aggregates, since they are much larger than the sizes detected by SANS. We suggest that the large aggregates are due to intermicellar entities formed for these dendrons that show micellar structures by SANS. However, for dendrons not exhibiting micellar structures by SANS, i.e. alkyl lengths of C12 and below, the association process is probably due to the formation of more random assemblies. Our conjecture is that the intermicellar structures are partly held together by electrostatic forces between the positively charged amine groups on the lysine heads and negatively charged trifluoroacetate present in the solution (the dendrons were synthesized as trifluoroacetate salts, Scheme 1). A more detailed discussion about the formation of micelles and intermicellar associations is given below.

In addition, the temperature effect was examined for some G1 dendrons (e.g. G1-C8, G1-C16 and G1(acetyl)-C16) and G2 dendrons (e.g. G2-C12 and G2-C16) showing no influence on the selfassembly, as illustrated for G1-C16 and G2-C16 in Fig. 7 at a solute concentration of $18 \mathrm{mM}$. To compensate for simple temperatureinduced changes in solvent viscosity, the first-order electric
A

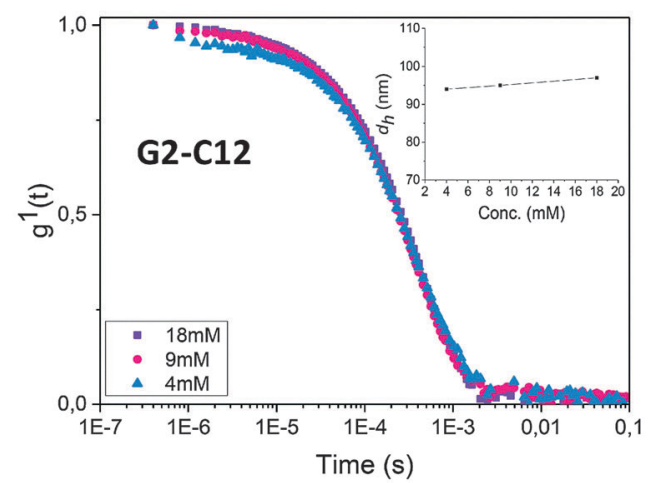

B

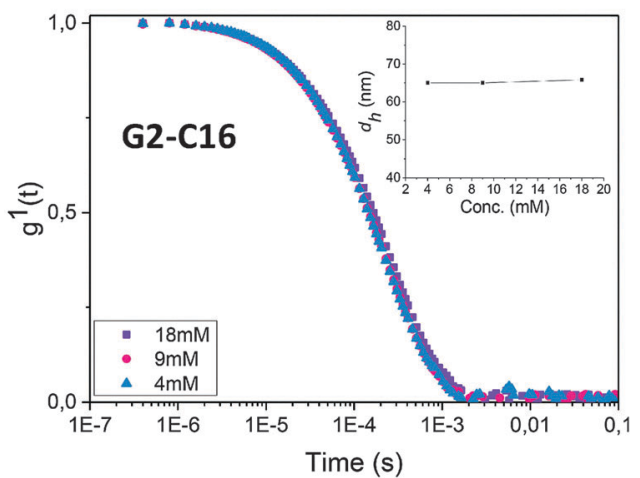

Fig. 6 First-order electric field autocorrelation function versus time obtained for (A) G2-C12 and (B) G2-C16 for different concentrations at $25{ }^{\circ} \mathrm{C}$ and $\mathrm{pH}$ ranging from 3 to 5 . The insets show the effect of concentration on the hydrodynamic radius.

field autocorrelation function, $g^{1}(t)$, was plotted against $t \cdot T / \eta$ (where $t$ is the time, $T$ is the absolute solution temperature, and $\eta$ is the solvent viscosity). Since fairly high temperatures are considered and no influence of temperature is detected on the decay of the correlation function, the results indicate that hydrogen bonds should not play an important role in the selfassembling behavior of these dendrons since hydrogen bonds are expected to be disrupted at elevated temperatures. This effect would lead to faster decay of the correlation function and therefore smaller size of the entities. Since neither solute concentration nor temperature seems to affect the self-assembling process and the species are stable over time, the formation of these very large supramolecular structures appears to be controlled by a mechanism that is not directly related to the conventional hydrogen and hydrophobic interactions. Another interesting feature observed in Fig. 7 is that for the G2-C16 sample the correlation functions at different temperatures can be portrayed by a single stretched exponential with $d_{\mathrm{h}}=65 \mathrm{~nm}$ and a value of $\beta=0.8$, which indicates fairly monodisperse entities. In contrast, for the G1-C16 sample the correlation functions reveal a bimodal relaxation mode that can be well described by eqn (4). In this case, micelles $\left(d_{\mathrm{hf}}=6 \mathrm{~nm}\right)$ coexist with intermicellar structures $\left(d_{\mathrm{hs}}=88 \mathrm{~nm}\right)$. The reason for this difference in the self-assembling behavior of the dendrons is not clear, but it seems that the bulky G2-C16 dendrons are more inclined 
A

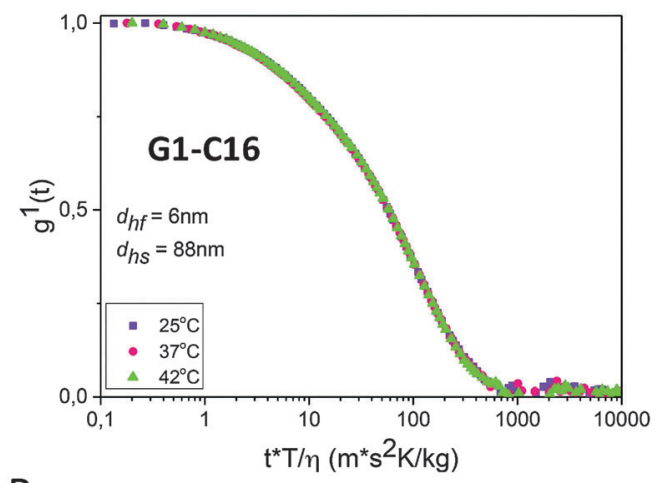

B

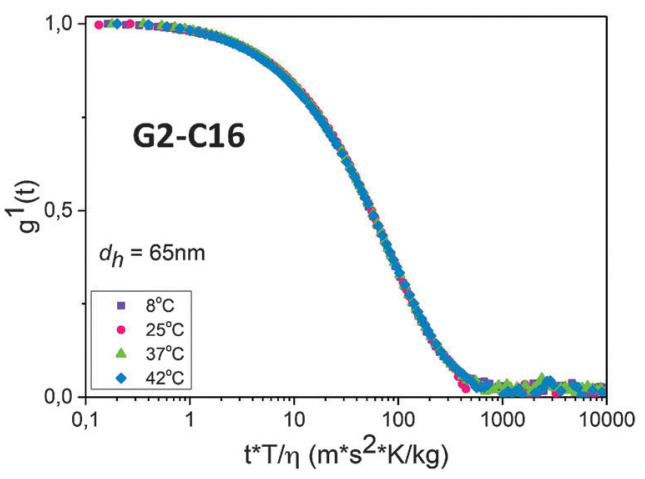

Fig. 7 First-order electric field autocorrelation function at a scattering angle of $124^{\circ}$ vs. $t \cdot T / \eta$ obtained for (A) G1-C16 and (B) G2-C16 at different temperatures and at a solute concentration of $18 \mathrm{mM}$.

to form intermicellar structures. It is interesting to note that the lower value of $d_{\mathrm{hf}}$ around $6 \mathrm{~nm}$ corresponds well with the sizes found by SANS, i.e. a radius of 2.5-2.9 $\mathrm{nm}$ (depending on the dendron type, cf. Tables 2-4), giving a diameter of 4.9 to $5.8 \mathrm{~nm}$.

Since we did not observe any concentration effects, a concentration of $18 \mathrm{mM}$ was chosen for the analysis of the rest of the dendrons, besides G1-C16 and G1(acetyl)-C16, which were analyzed at a concentration of $27 \mathrm{mM}$. The DLS measurements indicate that a minimum C-terminal alkyl tail length of C8 was needed for the dendrons to form clusters, since no correlation functions were obtained with dendrons having tail lengths below C8 (Fig. 8A).

Surprisingly, no self-assembly was observed for G1-C12 by DLS, even though the corresponding dendrons with shorter alkyl tail groups were showing association behavior. Zeta potentials were determined for the different dendrons and ranged from 11 to $55 \mathrm{mV}$. However, there was no clear correlation between zeta potential values and the ability of the dendrons to self-assemble. ${ }^{28,29}$ The hydrodynamic diameters $d_{\mathrm{h}}$ of the aggregates obtained by DLS for G1-C8/C14/C16/C18, G1(acetyl)-C10/C16 and G2-C12/C16 ranged from approximately 65 to $370 \mathrm{~nm}$ showing the formation of large interchain architectures. ${ }^{30}$ It is obvious from Fig. 8A that the correlation function for the G1-C14 dendron exhibits the most pronounced bimodal appearance and this behavior supports the coexistence of micelles and intermicellar structures.
A

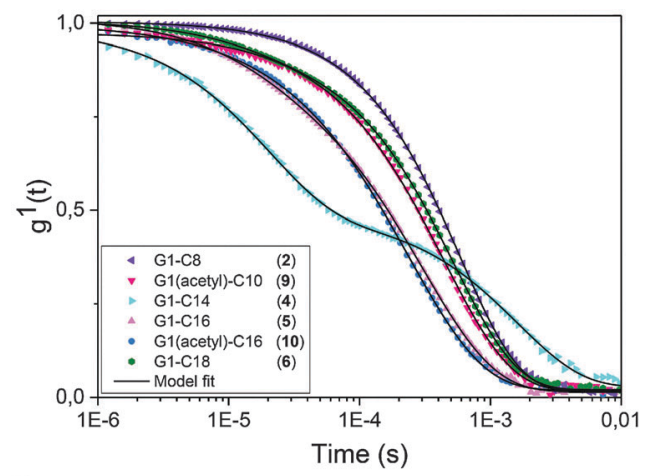

B

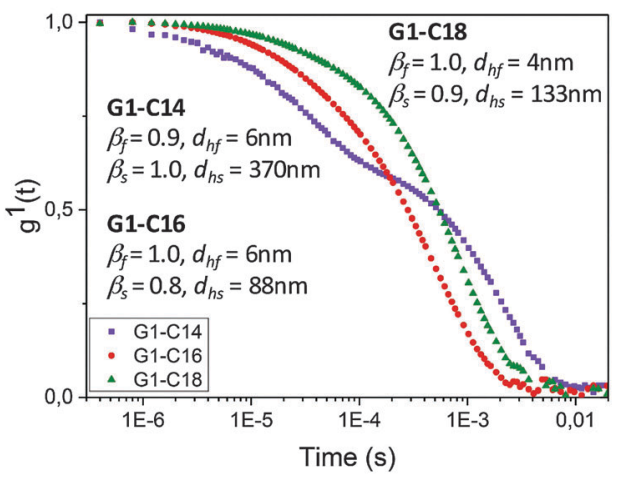

Fig. 8 (A) First-order electric field autocorrelation functions at a scattering angle of $124^{\circ}$ obtained for G1 dendrons with a tail length equal to or above C8. Only G1-C14, G1-C16 and G1-C18 exhibit a bimodal behavior and the other systems exhibit unimodal decay. (B) First-order electric field autocorrelation functions versus time for G1-C14, G1-C16, and G1-C18 systems at a scattering angle of $90^{\circ}$ and a temperature of $25^{\circ} \mathrm{C}$.

The stretched exponential $\beta$ characterizes the width of the distribution of relaxation times and $\beta$ is 1.0 for a single exponential. In this study $\beta$ is in the range $0.8-1.0$ showing a fairly narrow size distribution. In Fig. $8 \mathrm{~B}, g^{1}(q, t)$ is plotted versus time for the G1-C14, G1-C16, and G1-C18 samples and both the fast and slow relaxation modes are diffusive. The appearance of two relaxation modes for these samples can probably be attributed to increased hydrophobicity due to the long alkyl chains in these dendrons. This feature should make them more inclined to form intermicellar structures. The bimodal profile of the correlation functions is most pronounced for the G1-C14 system, but the bimodal appearance can also be established for the other displayed systems. The results disclose the coexistence of micelles $\left(d_{\mathrm{hf}} \approx 4-6 \mathrm{~nm}\right)$ and large intermicellar species. The sizes of the micelles from DLS are close to the values observed for the same systems by SANS. The largest hydrodynamic diameter $\left(d_{\mathrm{hs}}=370 \mathrm{~nm}\right)$ is observed for the G1-C14 sample, whereas the smallest $\left(d_{\mathrm{hs}}=88 \mathrm{~nm}\right)$ is found for the G1-C16 system. It seems that the sample with the shortest carbon chain is more inclined to form large interchain structures. When the alkyl chain is longer, the effect of steric hindrance may come into play and this may prevent the formation of large intermicellar associations.

The hydrodynamic diameters obtained from DLS are summarized in Fig. 9 for the systems indicated in the figure. Fig. 9A 
reveals that only the G1-C14/C16/C18 dendrons show a bimodal behavior with a fast and a slow relaxation mode. The value of $d_{\mathrm{hs}}$ in Fig. 9A shows a maximum for the G1-C14 dendron and this suggests that this system is inclined to form large associated structures. The dendrons with longer alkyl chains (C16 and C18) seem to form smaller aggregates. The values of $d_{\mathrm{hf}}$ from the fast relaxation mode do not give a specific trend. In spite of the fact that SANS data for the G1(acetyl)-C16 dendron system suggest the formation of well-developed micelles, we are not able to detect a fast relaxation mode in the correlation function, representing the micelles, for this system. The reason for this is attributed to the small amplitude of the fast mode, compared to the slow mode representing the intermicellar clusters. As a result, the fitting procedure is not able to catch the fast relaxation mode.

The results for the G2-C12 and G2-C16 dendrons show that the correlation functions can be described by a single relaxation mode (eqn (3)) and the value of $d_{\mathrm{h}}$ decreases as the alkyl chain becomes longer ( $c f$. Fig. 9B). The hypothesis for the absence of the fast mode (micelles) is that the larger head groups in the G2 dendrons can obstruct the formation of regular micelles and this conjecture is supported by the previous SANS results. The decrease of $d_{\mathrm{h}}$ may indicate that the packing efficiency inside the species may be augmented for longer alkyl chains.

\section{A}

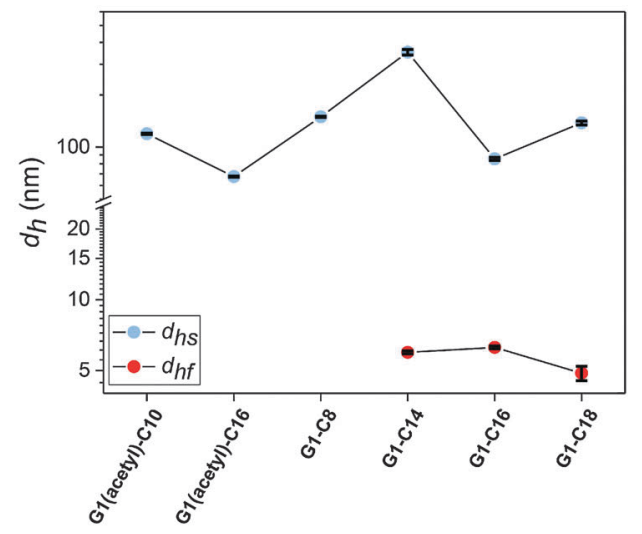

B

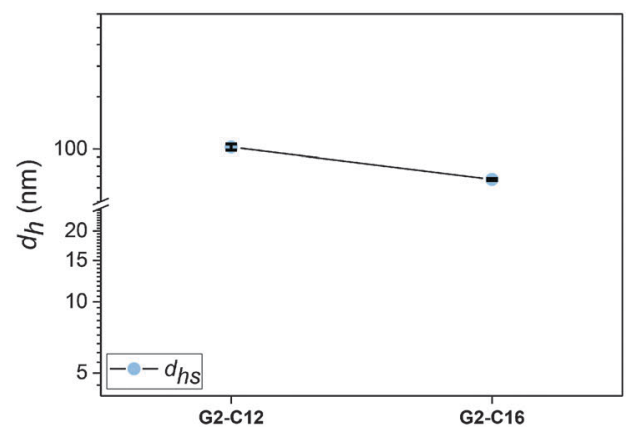

Fig. 9 The hydrodynamic diameter $\left(d_{\mathrm{h}}\right)$ obtained by DLS presented with error bars (approximately of the same size as the symbols) as a logarithmic function. (A) G1 dendrons with G1-C14/C16/C18 showing a bimodal behavior with the fast mode giving $d_{\mathrm{hf}}$ and the slow $d_{\mathrm{hs}}$. (B) G2 dendrons only showing unimodal behavior.
Next, we studied the $\mathrm{pH}$ effect on the sizes of the moieties formed by the dendrons. By examining the self-assembling ability of the G2-C16 dendron at pH 5, 7.4 and 9 in a phosphate buffer, we could conclude that an acidic $\mathrm{pH}$ was best suited to evoke self-assembly. The G2-C16 dendron precipitated at $\mathrm{pH}$ 7.4 and 9. However, at pH 5, the G2-C16 dendron was solvated showing increased size for the intermicellar aggregates giving a $d_{\mathrm{h}}$ value of $212 \mathrm{~nm}$, in comparison to the $d_{\mathrm{h}}$ value of $117 \mathrm{~nm}$ measured at pH 4 (Fig. 10). The observed pH effect confirmed our hypothesis that the formation of intermicellar aggregates could be due to electrostatic forces between micelles. At higher $\mathrm{pH}$ values these forces would not be effective due to the removal of the positive charge from the amines in the lysine head groups.

In Fig. 11, we have summarized our main findings in a schematic way, based on information obtained from both DLS and SANS data. As indicated in the figure, the length of the alkyl chain is an important parameter with respect to micelle formation. Micelles (with a diameter of approx. $5 \mathrm{~nm}$ ) were created only for chain lengths of $\mathrm{C} 14$ and above, and their core-shell structure could be described in detail by SANS. Below this length the alkyl chain is too short for micelle formation, since the total interaction energy is not sufficient to compensate for the bulky head group of the dendrons. However, due to their hydrophobic character, the alkyl chains will interact in other ways to form ill-defined aggregates. In addition to single dendrons (unimers), it is therefore likely that they also form dynamic irregular clusters with a size detected by DLS. A simplified image of such structures is illustrated in the upper right of Fig. 11 (for $\mathrm{C} \leq 12$ ). The alkyl chain is generally not expected to be straight when the dendron is in solution allowing different kinds of internal arrangements within such clusters. When the condition for micelle formation is satisfied (in terms of chain length, i.e. $\mathrm{C} \geq 14$ ), large intermicellar structures can exist, as schematically illustrated in the lower right of Fig. 11. These were found by DLS to be in the range of 65 to $370 \mathrm{~nm}$. In the intermicellization process sticky micelles collide and they form intermicellar aggregates. Due to the size limitation of SANS (ca. $70 \mathrm{~nm}$, as indicated at the bottom of Fig. 11), these

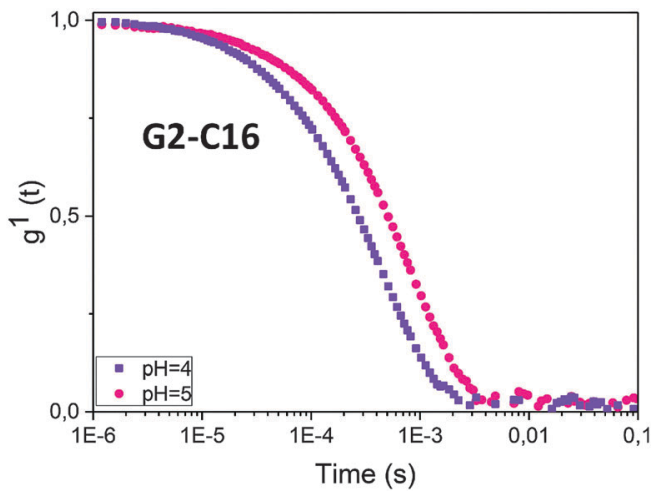

Fig. 10 First-order electric field autocorrelation functions versus time for $\mathrm{G} 2-\mathrm{C} 16$ at $25^{\circ} \mathrm{C}$ and at the $\mathrm{pH}$ values indicated. A PBS buffer was used and it was adjusted to the considered $\mathrm{pH}$ values. 


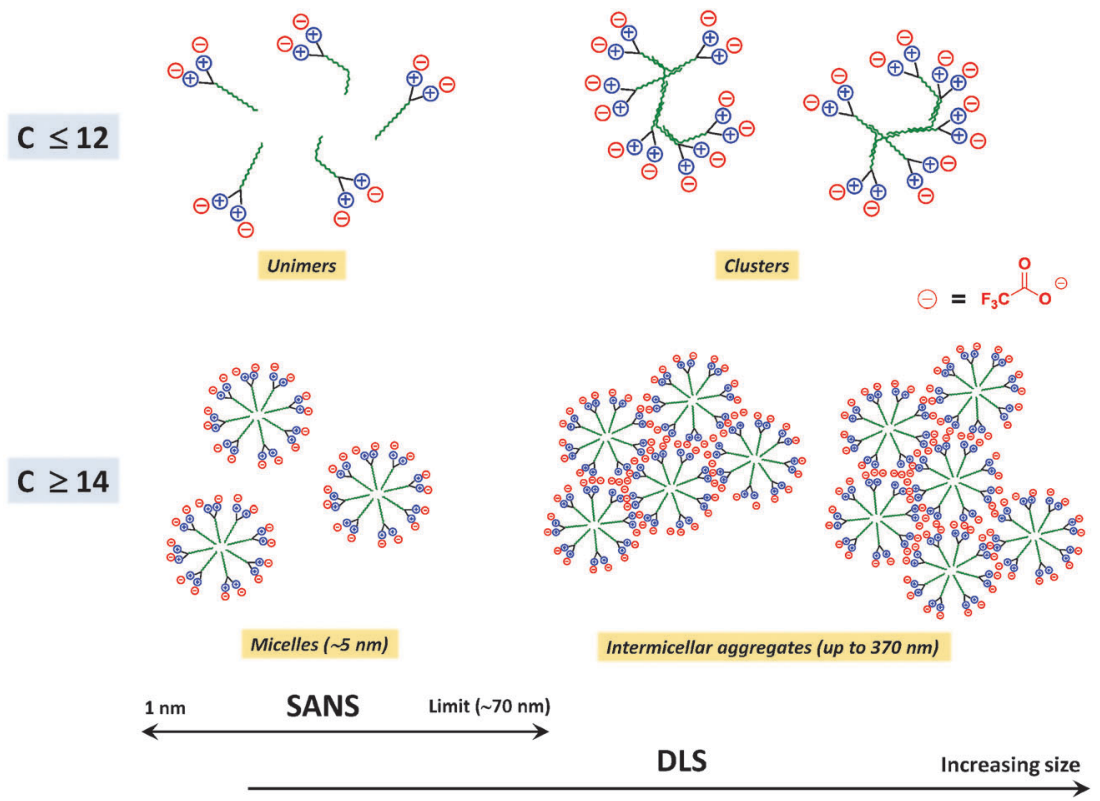

Fig. 11 Schematic view of micellar and intermicellar structures formed by dendrons in this study. For alkyl chain lengths of 12 carbons or less (C $\leq 12$ ), micelles are not formed, and the dendrons exist in solution as unimers and large irregular clusters, where the latter are detected by DLS. For alkyl chain lengths of 14 carbons or more $(C \geq 14)$, micelles are formed with a core-shell structure as detailed by SANS, and with an overall diameter of approx. $5 \mathrm{~nm}$. For these alkyl lengths, large intermicellar structures also exist. These are not detectable by SANS due to the upper SANS-limit of approx. $70 \mathrm{~nm}$, but were followed by DLS.

structures are generally not seen by SANS, apart from occasional signs of a tail in the SANS scattering pattern at the lowest $q$-values.

\section{Cell viability}

To explore the biocompatibility of dendrons, they were subjected to in vitro cytotoxicity testing on mouse fibroblast (NIH/3T3) and human embryonic kidney (HEK 293T) cells. The cytotoxicity in $\mathrm{NIH} / 3 \mathrm{~T} 3$ cells was examined in this study, because they are the most common cells used in cell experiments as models for connective tissues in the body. NIH/3T3 cells have a number of properties that make them attractive as cell culture models. They are hypertriploid and relative to other primary explant cultures, they are easy to establish and maintain. They proliferate rapidly and as a result large numbers of cells can be produced within several days. In addition, we have chosen HEK 293T as a chemical sensitive cell line model. This model was chosen because the kidney is a major site of organ damage caused by drug toxicity. MTS [3-(4,5-dimethylthiazol-2-yl)-5-(3-carboxymethoxyphenyl)-2-(4-sulfophenyl)-2 $H$-tetrazolium] assay was used as a colorimetric assay to evaluate cell viability measured as the quantity of formazan product ${ }^{31}$ after treating the cells with dendrons at three different concentrations (5, 10 and $20 \mu \mathrm{M})$. Chosen concentrations were inspired from the results published by Dong Ma et al. ${ }^{32}$ Since Dong Ma and co-workers did not observe any cytotoxicity for a G2 polylysine dendron cyclodextrin conjugate at concentrations up to $6.3 \mu \mathrm{M}\left(50 \mu \mathrm{g} \mathrm{mL}{ }^{-1}\right)$ we chose $5 \mu \mathrm{M}$ as the lowest concentration for our much smaller dendrons and increased 4-fold to obtain $20 \mu \mathrm{M}$ as the highest concentration (corresponding to $8-23 \mu \mathrm{g} \mathrm{mL}^{-1}$ ).
We expected the G1 dendrons with four free amine groups to show an increased cytotoxicity relative to $\mathrm{G} 1$ dendrons that were partially acetylated (two free amine groups), since it is known that positively charged amine groups are toxic to cells. ${ }^{9,33}$ Additionally, the cytotoxicity was expected to rise with higher concentrations. However, as seen in Fig. 12A and B, this is not the case. The two types of G1 dendrons exhibit similar non-cytotoxic behavior with increasing concentration.

Furthermore, we wanted to study the effect on cytotoxicity for a G2 dendron. For this we examined the G2-C16 dendron. The G2 dendron was expected to show an increased cytotoxicity profile relative to the $\mathrm{G} 1$ dendrons due to a greater number of positively charged amine groups on the surface (G2 dendrons having eight amine groups relative to four present on G1 dendrons). However, also here we observed high cell viability (Fig. 12). Overall it can be concluded that the dendrons do not display cytotoxicity (cell viability $>80 \%$ ) at concentrations up to $20 \mu \mathrm{M}$.

\section{Conclusion}

A series of novel amphiphilic polylysine dendrons containing variable alkyl chain lengths were successfully prepared by solidphase synthesis using the backbone amide linker approach. From the obtained small angle neutron scattering (SANS) results it could be concluded that ordered micellar structures only formed for dendrons with alkyl chain lengths above C12. For dendrons with longer alkyl chains than C12, the SANS data could be adequately fitted by a spherical core-shell model, 
A

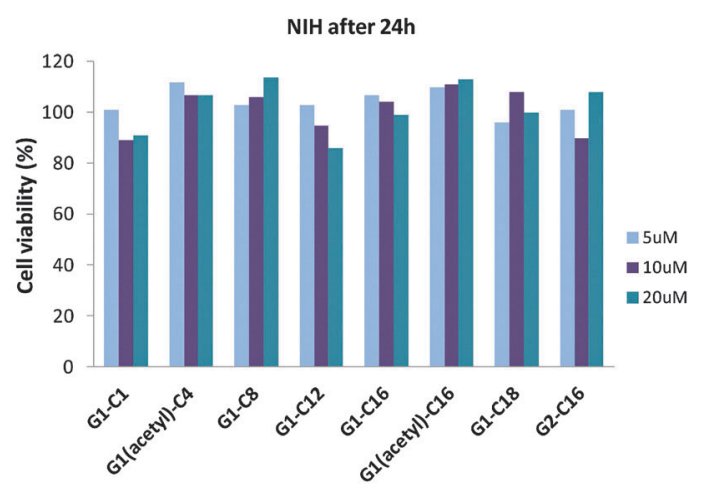

B

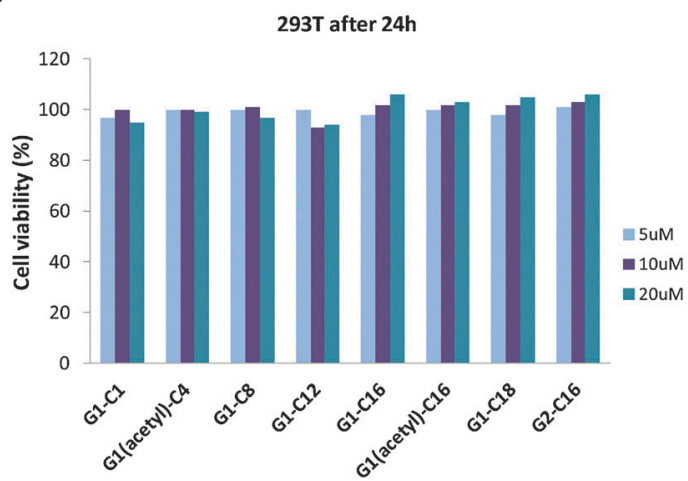

Fig. 12 Cell viability of $\mathrm{NIH} / 3 \mathrm{~T} 3$ (A) and HEK 293T (B) cells relative to untreated control cells of G1 and G2 dendrons at three different concentrations. The standard deviation was calculated to be less than 0.05 and 0.01 for NIH/3T3 and HEK 293T, respectively.

taking into account Coulombic interaction between the micelles. The overall size of the micellar structures formed by G1 dendrons was found to be around $5 \mathrm{~nm}$ corresponding well with the micellar diameter being spanned by two dendrons. Also, the number of dendrons per micellar structure in G1 dendrons, i.e. the aggregation number $N(\mathrm{agg})$, was estimated and ranged from 33 to 59 depending on the acetylation degree. Furthermore, it was observed that the bigger head groups in G2 dendrons may have a destabilizing effect on the micelle formation resulting in less regular micelles compared to the corresponding G1 dendrons. Based on this study, G1 dendrons with a minimum alkyl chain length of $\mathrm{C} 14$ would be best suited for the formation of stable micelles. Moreover, a modification of the head groups rendering them less positive would increase the micelle forming ability of the dendrons, but this must be balanced against the increased tendency for intermicellar aggregate formation when the surface charge is reduced. In addition to the micelle formation disclosed by the SANS and dynamic light scattering (DLS) results, the DLS experiments also revealed the presence of large intermicellar structures having sizes of $65-370 \mathrm{~nm}$.

The in vitro cytotoxicity of selected G1 and G2 dendrons was evaluated in mouse fibroblast (NIH/3T3) and human embryonic kidney (HEK 293T) cells by employing MTS [3-(4,5-dimethylthiazol-2-yl)-5-(3-carboxymethoxyphenyl)-2-(4-sulfophenyl)-2Htetrazolium] assay. Some level of cytotoxicity was expected due to the presence of positively charged amines on the lysine head groups. However, all dendrons display similar cytotoxicity profiles regardless of surface modification and generation number with no increased cytotoxicity at higher concentrations. Furthermore, there are no significant differences in cytotoxicity profiles when considering short versus long alkyl chain lengths. Given that the cell viabilities are well above $80-90 \%$, this indicates the possibility for further development of these dendritic systems for biomedical applications.

\section{Experimental section}

\section{Materials and methods}

All commercially available starting materials were purchased from Sigma-Aldrich, IRIS Biotech GmbH and Merck Chemicals and used without further purification. Solvents were purchased from Fischer Scientific and were of HPLC grade. Aminomethylated polystyrene resin (50-100 mesh size; $2 \mathrm{mmol} \mathrm{g}^{-1}$ loading; $1 \%$ crosslinked with divinylbenzene) was employed and the solid-phase reactions were performed in polypropylene syringes equipped with a polyethylene filter, or a glass container with a glass filter which was placed on a shaker. Washing of the resin was carried out manually on Torviq plates using $10 \mathrm{~mL}$ for each wash if not stated otherwise. After the reductive amination step, the resin was typically washed with $\mathrm{MeOH}(3 \times), \mathrm{NMP}$ and DCM $(15 \times), \mathrm{MeOH}(3 \times)$, NMP $(5 \times)$, DCM $(5 \times)$, and MeOH $(2 \times)$. Washing for the subsequent steps was typically performed with NMP and DCM $(10 \times)$ and $\mathrm{MeOH}(3 \times)$. Fmoc deprotection was typically carried out with piperidine/NMP $(1: 4,10 \mathrm{~mL})$ for $3+30+20 \mathrm{~min}$, or until ninhydrin test gave a positive read out. Dendrons were characterized by ${ }^{1} \mathrm{H}-\mathrm{NMR}(600 \mathrm{MHz}),{ }^{13} \mathrm{C}-\mathrm{NMR}(151 \mathrm{MHz}), \mathrm{COSY}\left({ }^{1} \mathrm{H}^{-1} \mathrm{H}\right.$ coupling), HSQC $\left({ }^{13} \mathrm{C}-{ }^{1} \mathrm{H}\right.$ coupling) and DEPT-135 ${ }^{13} \mathrm{C}-\mathrm{NMR}$ using a Bruker Ultrashield AVANCE or AVANCE II 600 with a cryo platform. Spectra were calibrated relative to the residual solvent peak $\left(\mathrm{D}_{2} \mathrm{O}\right.$ : $4.79 \mathrm{ppm}$ ). UPLC-MS analysis was conducted on an instrument comprising a Shimadzu Nexera X2 and a Bruker MicrOTOF-Q III, using a Phenomenex Kinetex C8 column $(2.6 \mu \mathrm{m}, 100 \AA$, $50 \times 2.1 \mathrm{~mm}$ ) or an Ascentis Express Peptide ES-C18 column

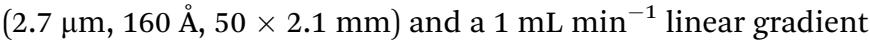
0-2.7 min (0-20\% buffer B), 2.7-6.0 min (20-100\% buffer B), 6.0-7.0 min (100-0\% buffer B), stop time $8.2 \mathrm{~min}$. Buffer A: $0.025 \%$ TFA in $10 \%$ aqueous MeCN. Buffer B: $0.025 \%$ TFA in $90 \%$ aqueous MeCN. Infrared spectroscopy (IR) was performed directly on the resin beads using a Shimadzu IR-affinity spectrometer based on the technique of attenuated total reflectance (ATR). The compound Lys-C16 was prepared according to the procedure published by Gallot et al. ${ }^{34}$

\section{Preparations}

FMPB derivatized $\operatorname{resin}^{15}$. Aminomethylated polystyrene resin ( 1 equivalent, $5 \mathrm{~g}$ ) was added to a filter syringe/glass container with a glass filter and swelled in NMP $\left(7.8 \mathrm{~mL} \mathrm{~g}^{-1}\right.$ resin). FMPB linker (1 equiv.), HBTU or PyBOP ( 0.95 equiv.) and DIPEA (1.9 equiv.) were added and the mixture was swirled for $24 \mathrm{~h}$ at rt and then washed with NMP $(15 \times 100 \mathrm{~mL})$ and DCM 
$(15 \times 100 \mathrm{~mL})$. The resin was capped with $\mathrm{Ac}_{2} \mathrm{O} / \mathrm{DIPEA} / \mathrm{DCM}$ $\left(10 / 5 / 85 ; 30 \mathrm{~mL} \mathrm{~g}^{-1}\right.$ resin) for $2-4 \mathrm{~h}$ at $\mathrm{rt}$. The derivatized resin was washed as above and additionally with $\mathrm{MeOH}(3 \times 100 \mathrm{~mL})$ followed by air-drying and drying in vacuo. Ninhydrin test (negative, yellow/pale grey supernatant) and DNPH test (positive, dark orange-red resin) were carried out.

Cleavage of the final dendron from the resin, procedure $A$. The derivatized resin was treated with TFA/DCM $(1: 1,10 \mathrm{~mL})$ and shaken for $2 \mathrm{~h}$, followed by filtration and collection of the cleavage mixture in a round bottomed flask. Evaporation in vacuo and precipitation in cold $\mathrm{Et}_{2} \mathrm{O}$ gave the product as a white powder.

G1 dendron with C-terminal C1, C8, C12, C14, C16 and C18 chains, procedure B. The FMPB-derivatized resin $(500 \mathrm{mg}$, 0.5 mmol, 1 equiv.), $\mathrm{NaBH}_{3} \mathrm{CN}$ ( $315 \mathrm{mg}, 5 \mathrm{mmol}, 10$ equiv.), and amine ( $5 \mathrm{mmol}, 10$ equiv.) were suspended in a $5 \% \mathrm{AcOH}$ in NMP solution $(6 \mathrm{~mL})$ in a $20 \mathrm{~mL}$ filter syringe and shaken. After $20 \mathrm{~h}$, the resin was washed followed by air-drying and drying in vacuo. DNPH test (negative, pale orange to yellow resin) and secondary amine test (positive, orange-brown resin) were carried out. Fmoc-Lys(Fmoc)-OH (1.48 g, 2.5 mmol, 5 equiv.) and DIC (0.20 mL, $1.25 \mathrm{mmol}, 2.5$ equivalents) were added to the filter syringe containing the derivatized resin and DCM/NMP $(95: 5,10 \mathrm{~mL})$. The suspension was mixed using a spatula until a waxy mass was obtained. After $20 \mathrm{~h}$ the resin was washed followed by air-drying and drying in vacuo. Secondary amine test (negative, colorless resin) was conducted. Fmoc removal was carried out followed by washing. Fmoc-Lys(Boc)-OH (937 mg, 2 mmol, 4 equiv), PyBOP ( $989 \mathrm{mg}, 2 \mathrm{mmol}, 3.8$ equiv.) and DIPEA ( $0.68 \mathrm{~mL}, 3.9 \mathrm{mmol}, 7.8$ equiv.) were transferred to the filter syringe containing the derivatized resin and NMP $(6 \mathrm{~mL})$ and shaken for $3 \mathrm{~h}$, or repeated until the ninhydrin test was negative. ${ }^{35}$ The resin was washed followed by Fmoc removal. Cleavage of the dendron was performed by procedure A.

Partially acetylated G1 dendron with C-terminal C4, C6, C10 and $\mathrm{C16}$ chains. Procedure B was followed; however, before the cleavage step the derivatized resin was treated with $\mathrm{Ac}_{2} \mathrm{O}$ / DIPEA/DCM (10 mL, 10/5/85) and shaken for $3 \mathrm{~h}$. The resin was washed followed by air-drying. Ninhydrin test (negative, yellow/pale grey supernatant) was carried out. Cleavage of the dendron was performed by procedure A.

G2 dendron with C-terminal C1, C12 and C16 chains, procedure C. The FMPB-derivatized resin $(500 \mathrm{mg}, 0.5 \mathrm{mmol}$, 1 equiv.), $\mathrm{NaBH}_{3} \mathrm{CN}$ (315 mg, 5 mmol, 10 equiv.) and amine ( 5 mmol, 10 equiv.) were suspended in a $5 \%$ AcOH in NMP solution $(6 \mathrm{~mL})$ in a $20 \mathrm{~mL}$ filter syringe and shaken. After $20 \mathrm{~h}$, the resin was washed followed by air-drying and drying in vacuo. DNPH test (negative, pale orange to yellow resin) and secondary amine test (positive, orange-brown resin) were carried out. Fmoc-Lys(Fmoc)-OH (1.48 g, 2.5 mmol, 5 equiv.) and DIC ( $0.20 \mathrm{~mL}, 1.25 \mathrm{mmol}, 2.5$ equiv. $)$ were added to the filter syringe containing the derivatized resin and DCM/NMP (95:5, $10 \mathrm{~mL}$ ). The suspension was mixed using a spatula creating a waxy mass. After $20 \mathrm{~h}$ the resin was washed followed by air-drying and drying in vacuo. Secondary amine test (negative, colorless resin) was conducted. Fmoc removal was carried out followed by washing. Fmoc-Lys(Fmoc)-OH (1.18 g, $2 \mathrm{mmol}$, 4 equiv.), PyBOP (989 mg, 2 mmol, 3.8 equiv.) and DIPEA ( $0.68 \mathrm{~mL}, 3.9 \mathrm{mmol}, 7.8$ equiv.) were added to the filter syringe containing the derivatized resin and NMP $(6 \mathrm{~mL})$ and shaken for $3 \mathrm{~h}$, or repeated until the ninhydrin test was negative. The resin was washed followed by Fmoc deprotection. Fmoc-Lys(Boc)-OH (937 mg, 2 mmol, 4 equiv.), PyBOP (989 mg, 2 mmol, 3.8 equiv.) and DIPEA (0.68 mL, $3.9 \mathrm{mmol}, 7.8$ equiv.) were transferred to the filter syringe containing the derivatized resin and NMP (6 mL) and shaken for $3 \mathrm{~h}$, or repeated until the ninhydrin test was negative. The resin was washed followed by Fmoc removal. Cleavage of the dendron was performed by procedure $\mathrm{A}$.

\section{Physicochemical measurements}

Small angle neutron scattering. The SANS instrument at the 2MW JEEP II reactor at the Institute for Energy and Technology (IFE) at Kjeller (Norway) was employed for the measurements. A velocity selector (Dornier) was used, with a wavelength spread of $\Delta \lambda / \lambda=10 \%$. Two different sample detector distances $(1.0 \mathrm{~m}$ and $3.4 \mathrm{~m}$ ) and two different neutron wavelengths $(5.1 \AA$ and $10.2 \AA)$ were used in order to obtain a total scattering range (q-range) from $0.008 \AA^{-1}$ to $0.25 \AA^{-1}$ corresponding to an observable particle size range of $2.5-78.5 \mathrm{~nm}$. The neutron detector was a circular $128 \times 128$ pixel ${ }^{3} \mathrm{He}$-filled RIS $\emptyset$ type inside a shielded detector chamber with vacuum to reduce air scattering. All samples were dissolved in $\mathrm{D}_{2} \mathrm{O}$ to reduce incoherent scattering and maximize the scattering contrast. The samples were inspected before being introduced to $2 \mathrm{~mm}$ quartz cuvettes and placed in the sample chamber. The transmission was measured separately and the scattering was normalized to absolute units $\left(\mathrm{cm}^{-1}\right)$ by taking into account data from the empty cell, the beam without cell and blocked-beam background scattering for data reduction. Chosen dendron samples were measured and all measurements were performed at room temperature.

Dynamic light scattering. DLS experiments were carried out using an ALV/CGS-8F goniometer system with 8 fiber-optical detection units, from ALV-GmbH, Langen, Germany. The intensity correlation functions were measured at 8 scattering angles simultaneously in the range $22-141^{\circ}$. The beam from a Uniphase cylindrical $22 \mathrm{~mW}$ HeNe-laser, operating at a wavelength of $632.8 \mathrm{~nm}$ with vertically polarized light, was focused on the sample cell (10 mm NMR tubes, Wilmad Glass Co., of highest quality) through a temperature-controlled cylindrical quartz container (with 2 plane-parallel windows), which was filled with a refractive index matching liquid (cis-decalin). The temperature in the container was controlled to within $\pm 0.01{ }^{\circ} \mathrm{C}$ with a heating/ cooling circulator. The samples were dissolved in MilliQ water and filtered in an atmosphere of filtered air through $0.45 \mu \mathrm{m}$ filters directly into pre-cleaned and dust free $10 \mathrm{~mm}$ NMR tubes.

Zeta potential and $\mathbf{p H}$ measurements. All zeta potential measurements were performed at $25{ }^{\circ} \mathrm{C}$ on a Zetasizer Nano ZS (ZEN3600, Malvern Instruments Ltd) using a "dip” cell with palladium electrodes. Laser Doppler micro-electrophoresis was employed to determine the electrophoretic mobility and automatically calculate the zeta potential from this value. 
Samples were prepared by dissolving the dendron samples in MilliQ water to give a final concentration of $18 \mathrm{mM}$ and $800 \mu \mathrm{L}$ was transferred to a PCS1115 cuvette to be placed in the sample chamber. Five to ten measurements were made for each sample and an average was taken for the zeta potential.

An InLab Micro $\mathrm{pH}$ electrode from Mettler Toledo made of glass (3 $\mathrm{M} \mathrm{KCl}$ as reference electrolyte) was employed for $\mathrm{pH}$ measurements. The $\mathrm{pH}$ values were measured at room temperature on $18 \mathrm{mM}$ solutions of dendrons in MilliQ water.

Cell viability. In this study cytotoxicity assay was carried out by using NIH/3T3 as a standard mouse fibroblast cell line and the human embryonic kidney cell line, HEK 293T, as a chemical sensitive model. The kidney model was used because the kidney is a major site of organ damage caused by drug toxicity. ${ }^{36-38}$

NIH/3T3 and HEK 293T were purchased from ATCC (American Type Culture Collection) and cultured in T75 cell culture flasks in Dulbecco's modified Eagle's cell culture medium (DMEM) containing $10 \%$ fetal bovine serum (FBS) and 1\% penicillin-streptomycin (Sigma Aldrich). The monolayer was then trypsinized and the cells were seeded in 96-well plates at a density of $6 \times 10^{3}$ cells per well (100 $\mu \mathrm{L}$ per well) in the culture medium. Following $24 \mathrm{~h}$ incubation and attachment, the cells were treated with dendrons with concentrations of 5, 10 and $20 \mu \mathrm{M}$ and incubated for an additional $24 \mathrm{~h}$. Cell viability of cells treated with dendrons was evaluated using MTS assay as a colorimetric assay in solution with an electron coupling reagent, phenazine ethosulfate (CellTiter 96 AQueous One Solution Cell Proliferation Assay, Promega Corporation). $20 \mu \mathrm{L}$ MTS was added to each well and incubated for $2 \mathrm{~h}$ following the $24 \mathrm{~h}$ incubation with dendrons. The absorbance of the soluble formazan product was quantified by measuring the absorbance at $490 \mathrm{~nm}$ using a plate reader (Synergy Mx-Monochromator-based Multi-mode Microplate Reader, BioTek Instruments, Vermont, USA). The experiment was repeated four times for each group and relative cell viability was calculated as below:

$$
\text { Relative cell viability }(\%)=\frac{\left(A_{\text {sample }}-A_{\text {ref }}\right)}{A_{\text {control }}-A_{\text {ref }}} \times 100
$$

where $A_{\text {sample }}$ is the absorbance obtained from the wells containing dendron-treated cells, $A_{\text {ref }}$ the absorbance of blank medium $(100 \mu \mathrm{L}$ medium $+100 \mu \mathrm{L}$ MilliQ $+20 \mu \mathrm{L}$ MTS) and $A_{\text {control }}$ the absorbance of control cells (untreated cells).

\section{Acknowledgements}

B. N. gratefully acknowledges a grant from the Norwegian Research Council, SYNKNØYT for the project with the number 8411/F50. S. M. acknowledges Sandra Medel for assisting in the collection of DEPT-135 ${ }^{13} \mathrm{C}-\mathrm{NMR}$ data.

\section{References}

1 E. Buhleier, W. Wehner and F. Vögtle, Synthesis, 1978, 155-158.

2 D. A. Tomalia, H. Baker, J. R. Dewald, M. Hall, G. Kallos, S. Martin, J. Roeck, J. Ryder and P. Smith, Polym. J., 1985, 17, 117-132.
3 G. R. Newkome, Z. Q. Yao, G. R. Baker and V. K. Gupta, J. Org. Chem., 1985, 50, 2003-2006.

4 R. G. Denkewalter, J. Kolc and W. J. Lukasavage, US pat., 4 289 872, 1981.

5 R. G. Denkewalter, J. Kolc and W. J. Lukasavage, US pat., 4 410 688, 1983.

6 K. Sadler and J. P. Tam, Rev. Mol. Biotechnol., 2002, 90, 195-229.

7 J. P. Tam, Proc. Natl. Acad. Sci. U. S. A., 1988, 85, 5409-5413.

8 D. N. Prosnett, H. McGrath and J. P. Tam, J. Biol. Chem., 1988, 263, 1719-1725.

9 D. A. Tomalia, J. B. Christensen and U. Boas, Dendrimers, Dendrons and Dendritic Polymers, Cambridge University Press, 2012.

10 U. Boas, J. B. Christensen and P. M. H. Heegaard, Dendrimers in Medicine and Biotechnology, RSC Publishing, 2006.

11 M. Malkoch, E. Malmström and A. M. Nyström, Polymer Science: A Comprehensive Reference, 2012, 6, 113-175.

12 T. Yu, X. Liu, A.-L. Bolcato-Bellemin, Y. Wang, C. Liu, P. Erbacher, F. Qu, P. Rocchi, J.-P. Behr and L. Peng, Angew. Chem., Int. Ed., 2012, 51, 8478-8484.

13 X. Liu, J. Zhou, T. Yu, C. Chen, Q. Cheng, K. Sengupta, Y. Huang, H. Li, C. Liu, Y. Wang, P. Posocco, M. Wang, Q. Cui, S. Giorgio, M. Fermeglia, F. Qu, S. Pricl, Y. Shi, Z. Liang, P. Rocchi, J. J. Rossi and L. Peng, Angew. Chem., 2014, 126, 12016-12021.

14 X. Liu, C. Liu, J. Zhou, C. Chen, F. Qu, J. J. Rossi, P. Rocchi and L. Peng, Nanoscale, 2015, 7, 3867-3875.

15 D. K. Svenssen, S. Mirsharghi and U. Boas, Tetrahedron Lett., 2014, 55, 3942-3945.

16 U. Boas, J. Brask and K. J. Jensen, Chem. Rev., 2009, 109, 2092-2118.

17 S. K. Shannon and G. Barany, J. Comb. Chem., 2004, 6, 165-170.

18 See selected IR data in ESI $\dagger$.

19 The test solution was prepared by dissolving Fmoc-phenylalanine $(3 \mathrm{mg})$ in $2 \%(\mathrm{v} / \mathrm{v})$ acetaldehyde in NMP $(1 \mathrm{~mL})$. A few resin beads were put in a test tube and two drops of the test solution was added. After 3-5 minutes at room temperature, the formation of orange-brown resin beads indicates the presence of a secondary amine.

20 U. Boas and S. Mirsharghi, Org. Lett., 2014, 16, 5918-5921.

21 E. Kaiser, R. L. Colescott, C. D. Bossinger and P. I. Cook, Anal. Biochem., 1970, 34, 595-598.

22 S. Yokoyama and J.-I. Hiramatsu, J. Biosci. Bioeng., 2003, 95, 204-205.

23 See fully assigned NMR data on all compounds in ESI $\dagger$.

24 A. Guinier and G. Fournet, Small-Angle Scattering of X-Rays, 1955.

25 J. B. Hayter and J. Penfold, Mol. Phys., 1981, 42, 109-118.

26 N. J. Turro and A. Yekta, J. Am. Chem. Soc., 1978, 100, 5951-5952.

27 A. J. F. Siegert, Rad. Lab. Rep., No 465, Massachusetts Institute of Technology, 1943.

28 See Table $\mathrm{S} 1$ in ESI $\dagger$ for zeta potentials.

29 The zeta potential is a measure of the charge density of the particles in solution. Generally, solutions or suspensions of 
particles with a zeta potential at or above $\pm 30 \mathrm{mV}$ are considered electrostatically stable, whereas at lower values flocculation may occur.

30 The length of a $\mathrm{C}-\mathrm{C}$ bond is $0.154 \mathrm{~nm}$. Taking into account that the bonds are in a zig-zag pattern with respect to the main axis of the alkyl chain, the projection of the chain can be calculated to be 0.8 and $1.8 \mathrm{~nm}$ for a C8 and C18 chain, respectively. Assuming that the chain is fully stretched and that the lysine head groups in a G1 and G2 dendron are roughly 1-2 nm, the dendron length will be roughly 1.8-3.8 nm. If the micellar diameter is spanned by two dendrons this would give a diameter in the range of $3.6-7.6 \mathrm{~nm}$, i.e. well below $10 \mathrm{~nm}$. Hence, obtained sizes ranging from 65 to $370 \mathrm{~nm}$ must be due to intermicellar aggregates.

31 The MTS assay is an enzyme-based colorimetric method for cell viability assessment. In the mitochondria of metabolic active (living) cells MTS is reduced to a colored formazan product. Therefore, the quantity of formazan measured by the absorbance at $490 \mathrm{~nm}$ is directly proportional to the number of living cells in the culture.

32 D. Ma, H.-B. Zhang, Y.-Y. Chen, J.-T. Lin and L.-M. Zhang, J. Colloid Interface Sci., 2013, 405, 305-311.

33 K. Jain, P. Kesharwani, U. Gupta and N. K. Jain, Int. J. Pharm., 2010, 394, 122-142.

34 B. Gallot and H. H. Haj, Mol. Cryst. Liq. Cryst., 1989, 170, 195-214.

35 The liability of the ninhydrin test was low when synthesizing dendron derivatives on resin with alkyl chain lengths above C12 leading to false negative ninhydrin read-outs.

36 G.-E. Séralini, J. S. de Vendômois J, D. Cellier, C. Sultan, M. Buiatti, L. Gallagher, M. Antoniou and K. R. Dronamraju, Int. J. Biol. Sci., 2009, 5, 438-443.

37 R. Mesnage, E. Clair, S. Gress, C. Then, A. Székács and G.-E. Séralini, J. Appl. Toxicol., 2013, 33, 695-699.

38 G.-E. Séralini, R. Mesnage R, E. Clair, S. Gress, D. Cellier and J. S. de Vendômois, Environ. Sci. Eur., 2011, 23, 10. 